\title{
Design concepts for broadband high-efficiency DOES
}

\author{
B. H. Kleemann \\ b.kleemann@zeiss.de \\ M. Seeßelberg
}

J. Ruoff

\author{
Carl Zeiss AG, Corporate Research and Technology, Oberkochen, 73446, Germany \\ Carl Zeiss AG, Corporate Research and Technology, Oberkochen, 73446, Germany
}

Several design-concepts are presented for so-called efficiency achromatized diffractive optical elements (EA-DOEs) possessing a diffraction efficiency larger than $97 \%$ over a broad spectral range. We start with tracing two different methods for surface relief profiles well known from the literature: common depth and multilayer EA-DOEs. Successively we present the following new approaches together with design parameters and performance properties: 1) gradient-index EA-DOES, 2) sub-wavelength EA-DOEs, and 3) a so-called cut-and-paste strategy. All designs are based on scalar assumptions and certain necessary dispersion relations of two different materials. The scalar assumption is no real limitation as the minimum zone width of our main application, the correction of chromatic aberrations, is $50 \ldots 100$ times the wavelength. From aforementioned relations, design parameters as profile heights are derived and the resulting diffraction efficiency can be deduced. Moreover, for the multilayer and for the GRIN EA-DOEs we are able to show that if the dispersion relations of the materials can be accurately described by second order Cauchy series, the efficiency becomes generic and will be the same regardless of which materials are chosen. [DOI: 10.2971/jeos.2008.08015]

Keywords: diffractive optical element, achromatization, broadband, high efficiency

\section{INTRODUCTION}

By achromatization one mainly understands the correction of longitudinal chromatic aberrations in optical systems, which is due to the fact that at different wavelengths the glass materials possess different refractive indices, a phenomenon called dispersion. The longitudinal achromatization condition for two lenses 1 and 2 of different materials relates focal lengths $f_{1}, f_{2}$ and the corresponding Abbe numbers $v_{1}, v_{2}$ :

$$
\frac{1}{f_{1} v_{1}}+\frac{1}{f_{2} v_{2}}=0 .
$$

Since the Abbe numbers of glasses always have positive values between 20 and 90, in purely refractive optical systems longitudinal chromatic aberrations can only be corrected by using lenses with positive and negative focal lengths. This has been done in optical systems called dichromats, achromats, trichromats, or apochromats, where for two or three wavelengths, longitudinal chromatic aberrations have been eliminated. Between these wavelengths only a small secondary or tertiary spectrum remains.

It is possible to define a dispersion relation for diffractive optical elements as well. In what follows, the term DOE is used for diffractive lenses, gratings, holographic optical elements, computer generated holograms, and other kinds of diffractive optical elements with blazing phase. A DOE has a negative Abbe number $v_{\mathrm{DOE}} \approx-3.45$ (cf. e.g. [1]) if it is used for chromatic aberration correction in the visible spectrum. Because of the negative sign, the modified achromatization condition in Eq. (1) with $v_{2}$ replaced by $v_{\mathrm{DOE}}$ can even be fulfilled in case that focal lengths of both refractive and diffractive lenses have the same algebraic sign, which offers new design possibilities [1].

Optical lens systems with DOEs are often called hybrid optical systems. A lens with a DOE on one of its sides is called a hybrid lens. Some examples of hybrid optical systems can be found in [1]-[5]. The chromatic correction capability of DOEs can in principle be easily exploited to correct large chromatic errors occuring in broadband optical systems by an adapted zone width polynomial. Unfortunately, the diffraction efficiency decreases for wavelengths $\lambda$ deviating from the design wavelength $\lambda_{0}$, which prevents from frequent use of DOEs in broadband optical systems. Consequently, DOE usage is mostly limited to applications with small spectral bandwidth. This well known drawback holds for all conventional DOEs made of a single material. However, high broadband efficiency can e.g. also be achieved with a single material using the dispersion properties generated by sub-wavelength structures [6] or, for the reflection case, by appropriately designing the band gaps of photonic crystal rod gratings [7].

The above deficiency of conventional DOEs has been overcome for surface relief DOEs with common depth [8] consisting of two different materials, which are combined in such a way that the dispersion of both materials compensate the wavelength dependency of the optical phase in a broader 
wavelength interval. This approach can be further improved by allowing the profiles of the two material to have different depths [9]-[12]. These approaches are presented in [13, 14] and are nicely explained in more detail in [15] together with a discussion of their different fabrication tolerances and the accuracy of the applied theoretical models. All these diffractive elements show high diffraction efficiencies above $97 \%$, some even exceeding $99 \%$ over a broad spectral range from $\lambda_{\text {short }}$ to $\lambda_{\text {long. }}$

In the previous literature [14, 15], these DOEs for broadband use have been referred to as achromatized DOEs (A-DOEs). However, the term "achromatization" of optical elements is already assigned to the correction of longitudinal and other chromatic aberrations as we have discussed above. Hence, to make the new broadband property of DOEs more distinguishable from the well known focal length correction we prefer to call these diffractive elements "efficiency achromatized DOEs" (EA-DOEs).

EA-DOEs have a broad application range since they can be applied in all optical systems where conventional DOEs for the correction of longitudinal chromatic aberrations as well as of the chromatic difference of magnification are already used. These can be photo lenses [11], Head Mounted Displays as e.g. the so-called Cinemizer [16], eyepieces [17], telescopes, binoculars, glasses, and so on. For several years, photographic lenses with embedded broadband high-efficiency DOE are commercially available from Canon [11] ("EF 70-300/4.5-6.7 DO IS USM" and "EF 400/4.0 DO IS USM").

Within this paper, we present several new design concepts for EA-DOEs using gradient-index materials, sub-wavelength structures, and a so-called cut-and-paste strategy. Generally, these EA-DOEs are appropriate to substitute for all conventional DOEs schematically shown in Figure 1 with local zone width $d$. However, for the proposed concepts it does not matter whether zone width $d$ varies over a radial position or is constant which would be the case for the DOE being an axicon. Of course, the DOE could also be a simple linear grating.

The paper is arranged as follows. In Section 2, properties of conventional surface relief DOEs and EA-DOEs are briefly reviewed as an introduction to this topic. In Section 2.3 conditions are given resulting in a generic fully material independent efficiency behaviour. In Section 3, gradient-index EADOEs together with their properties are introduced and some ideas for their fabrication are given. Section 4 presents subwavelength EA-DOEs together with several embodiments and properties. The cut-and-paste design in Section 5 is a further generalisation of surface relief EA-DOEs.

\section{STATE OF THE ART DOES}

\subsection{Conventional DOES}

Generally, all DOEs like conventional rotationally symmetrical DOEs (cf. Figure 1) or linear diffraction gratings diffract light into several orders. It is, however, possible to choose a profile such that nearly all of the incoming light with design

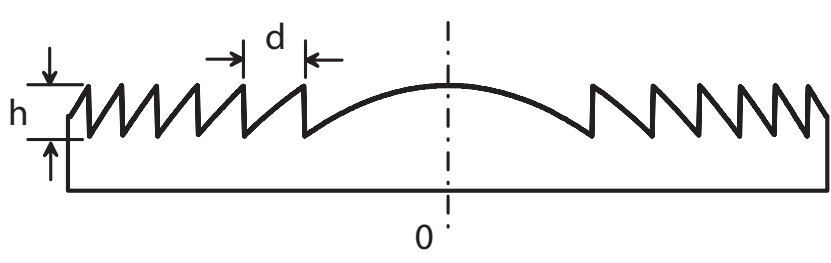

FIG. 1 Schematic representation of a cross section through a conventional DOE with sawtooth profiles of local zone width $d$ and profile depth $h$.

wavelength $\lambda_{0}$ is diffracted into a single diffraction order the blaze profile, which is a sawtooth profile of appropriate depth. If the DOE consists of a dielectric material with refractive index $n_{\mathrm{DOE}}$ and the superstrate material is air, then the optimum profile depth $h_{1}$ can be found from condition

$$
h_{1}=\frac{\lambda_{0}}{n_{\mathrm{DOE}}-n_{\mathrm{air}}}=\frac{\lambda_{0}}{n_{\mathrm{DOE}}-1} .
$$

Then the first order diffraction efficiency $\eta\left(\lambda_{0}\right)$ for normal incidence at the design wavelength $\lambda_{0}$ is $100 \%$ in scalar diffraction theory neglecting Fresnel reflection and all rigorous effects coming from finite thickness and profile edges of the DOE. For $100 \%$ efficiency in the $m$-th diffraction order the optimum profile depth $h_{1}$ has to be multiplied by $m$. However, without any loss of generality the present paper is restricted to the first diffraction order, i. e. $m=1$ which is most often used in practice. Oblique incidence is not considered here as it would go beyond the scope of this publication. For a detailed investigation concerning conventional blaze gratings, we refer to [18].

Deviations from the design wavelength $\lambda_{0}$ decrease the diffraction efficiency $\eta$ in the design order and may result in unwanted stray light (flare) and coloured double images coming from adjacent diffraction orders, if this light cannot be blocked by apertures or other constructive means. Generally, within scalar diffraction theory, the efficiency $\eta$ of the first diffraction order can be calculated from the phase function $\Phi(\lambda)$ according to the following relation [19], which is repeatedly required in the following:

$$
\eta(\lambda)=\operatorname{sinc}^{2}\left(\frac{\Phi(\lambda)}{2 \pi}-1\right) \text { with } \operatorname{sinc}(x):=\frac{\sin (\pi x)}{\pi x} .
$$

In the specific case of a surface relief blaze structure of a dielectric material adjacent to air, the diffraction efficiency of the design order as function of wavelength $\lambda$ and under consideration of the material dispersion can be calculated from Eq. (3) and the phase function

$$
\Phi(\lambda)=\frac{2 \pi \lambda_{0}}{\lambda} \frac{n(\lambda)-1}{n\left(\lambda_{0}\right)-1}
$$

Assuming the design wavelength to be $\lambda_{0}=550 \mathrm{~nm}$ for a DOE made of PMMA, the efficiency is only $55 \%$ at the lower limit of the visual spectrum at $\lambda_{\text {short }}=400 \mathrm{~nm}$ and about $85 \%$ at the upper limit of the visual spectrum at $\lambda_{\text {long }}=700 \mathrm{~nm}$. Calculations using rigorous diffraction theory [20,21] show a convergent behaviour as given in Figure 2 for grating peri- 
ods $d=10 \mu \mathrm{m}, d=30 \mu \mathrm{m}$, and $d=100 \mu \mathrm{m}$ at normal incidence for PMMA. Obviously, the differences between rigorous values for $d=100 \mu \mathrm{m}$ and scalar ones are hardly noticeable. The optimum depth $h_{1}=1.11 \mu \mathrm{m}$ according to Eq. (2) is determined for the refractive index $n=1.4936$ corresponding to PMMA at $\lambda_{0}$. Since scalar and rigorous efficiencies for large local zone widths $d$ agree so well, it is sufficient to use scalar efficiency in the following. Moreover, the minimum zone width of a DOE used for chromatic aberration correction is often as large as $d>50 \mu \mathrm{m}$ which justifies this decision.

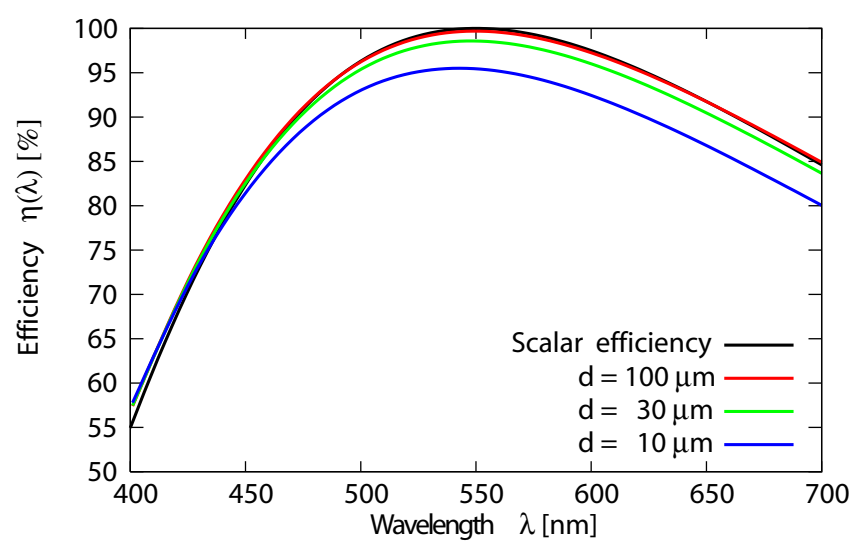

FIC. 2 Rigorous $1^{\text {st }}$ order diffraction efficiency of a surface relief blaze grating made of PMMA adjacent to air with grating periods $d=100 \mu \mathrm{m}, d=30 \mu \mathrm{m}$, and $d=10 \mu \mathrm{m}$ at normal incidence, design wavelength $\lambda_{0}=550 \mathrm{~nm}$ and with optimum depth $h_{1}=$ $1.11 \mu \mathrm{m}$. For comparison, scalar diffraction efficiency calculated from Eqs. (3), (4) is also given.

In order to reach $100 \%$ diffraction efficiency, the argument of the sinc-function has to be 0 , which leads to the condition

$$
\frac{\lambda_{0}}{\lambda} \frac{n(\lambda)-1}{n\left(\lambda_{0}\right)-1}=1 \Longleftrightarrow n(\lambda)=\frac{\lambda}{\lambda_{0}}\left(n\left(\lambda_{0}\right)-1\right)+1 .
$$

The above condition can only be fulfilled if $n(\lambda)$ is linearly increasing with increasing wavelength $\lambda$. However, all known real materials having normal dispersion show a monotonously decreasing dependence of the refractive index $n(\lambda)$ and are therefore not able to fulfill the above condition. This is the reason why the conventional DOE shown in Figure 1 cannot be an EA-DOE with a high broadband efficiency $\eta(\lambda)$.

\subsection{Common depth EA-DOEs}

Substituting the air between the sawtooths of the surface relief DOE profile in Figure 1 by a second material with another dispersion $n_{2}(\lambda)$ introduces an additional degree of freedom [8] and allows for dispersion compensation of the wavelength dependent optical phase in a specific wavelength interval. Schematically, so-called "common depth DOEs" look as shown in Figure 3. Without any restriction and throughout this paper, $n_{1}(\lambda)>n_{2}(\lambda)$ is assumed. Because of the dispersion of the second material, Eq. (4) for the phase function has now to be replaced by

$$
\Phi(\lambda)=\frac{2 \pi \lambda_{0}}{\lambda} \frac{n_{1}(\lambda)-n_{2}(\lambda)}{n_{1}\left(\lambda_{0}\right)-n_{2}\left(\lambda_{0}\right)},
$$

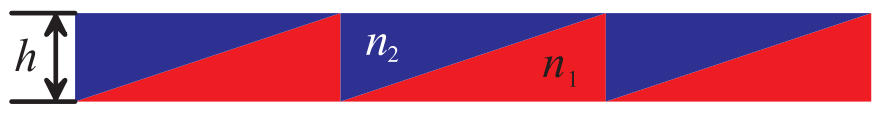

FIG. 3 Schematic representation of a DOE with common depth $h$, where the air between the sawtooths of a conventional blaze profile is substituted by a second material with another dispersion $n_{2}(\lambda)$.

with corresponding efficiency given by Eq. (3). Thus, condition in Eq. (5) becomes:

$$
\frac{\lambda_{0}}{\lambda} \frac{n_{1}(\lambda)-n_{2}(\lambda)}{n_{1}\left(\lambda_{0}\right)-n_{2}\left(\lambda_{0}\right)}=1
$$

which is equivalent to

$$
\Delta n(\lambda)=\frac{\lambda}{\lambda_{0}} \Delta n\left(\lambda_{0}\right) \quad \text { with } \quad \Delta n(\lambda)=n_{1}(\lambda)-n_{2}(\lambda) .
$$

Now, for high broadband efficiency, only the difference $\Delta n(\lambda)$ of the two refractive indices has to be linearly increasing with increasing wavelength $\lambda$. Although this condition is hardly fulfilled exactly, it is possible to find materials fulfilling Eq. (8) sufficiently well, resulting in high efficiency over a quite broad specific spectral range from $\lambda_{\text {short }}$ to $\lambda_{\text {long. }}$. An example for a common depth EA-DOEs consisting of polycarbonate and glass BaF52 is given in $[14,15]$, where dispersion curves and efficiency behaviour are given. Figures 12 and 13 show more examples of dispersion relations and efficiency curves for several material pairs suited to build common depth EA-DOEs.

\subsection{Multilayer EA-DOES}

Compared to common depth EA-DOEs, multilayer EA-DOEs $[10,11]$ allow for much more freedom in the choice of optical materials. As mentioned in the introduction, first examples of optical systems with built-in multilayer EA-DOE are commercially available which emphasizes the importance of this concept. This kind of EA-DOE is schematically represented in Figure 4 . Here, introducing a second profile depth by dividing the two materials by an air gap generates an additional degree of freedom.

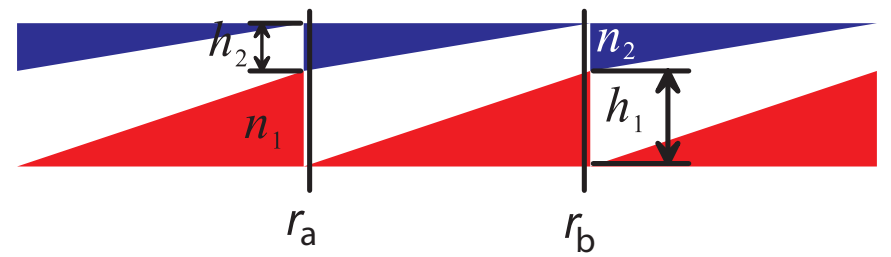

FIG. 4 Schematic representation of a multilayer DOE consisting of two sawtooth profiles of different materials together with paths in $r_{\mathrm{a}}$ and $r_{\mathrm{b}}$ at the boundaries of a local zone width $d=\left|r_{\mathrm{a}}-r_{\mathrm{b}}\right|$ to determine optical path difference. $r_{\mathrm{a}}$ and $r_{\mathrm{b}}$ denote positions located near both sides of the discontinuities of the sawtooth profiles.

In the following, we work out interesting properties of multilayer EA-DOEs, which apparently have not been published yet. We will show 1) that in principle any pair of different materials is suited to build a multilayer EA-DOE and 2) that efficiency is even independent of the materials if the refractive indices of both materials obey second order Cauchy relations. 
Simultaneously, these considerations correct misleading explanations of the functionality of a multilayer EA-DOE in [11] where it is regarded as a stack of two independent multi order DOEs.

The separation of both materials can, in principle, be arbitrary, as long as they are not too far apart from each other. The optical effect of all three embodiments of a multilayer DOE shown in Figure 5 is therefore the same. If, however, the gap becomes too large, the validity of the scalar diffraction theory is no longer guaranteed, and the multilayer DOE can no longer be considered as a single DOE, but rather as two independent ones, hence losing the assumed properties. In the following, the air gap is assumed to be small enough. As in the right embodiment of Figure 5, the second material can also be directly brought onto the first material [12]. This embodiment exhibits less critical fabrication tolerances. A discussion on how tolerances are affecting efficiency and on the applicability of different efficiency calculation methods can be found in $[14,15]$.

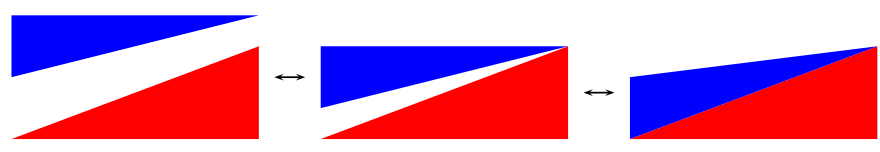

FIG. 5 Three optically equivalent embodiments of a multilayer EA-DOE.

Now, let us show the first characteristic that in principle, any pair of different materials with refractive indices given by $n_{1}(\lambda)$ and $n_{2}(\lambda)$ is suited to build a multilayer EA-DOE. According to Figure 4 , the depths of the two profiles are denoted by $h_{1}$ and $h_{2}$; the symbols $r_{\mathrm{a}}$ and $r_{\mathrm{b}}$ denote the left and right position of a local zone. Then, high diffraction efficiency $\eta$ of the first diffraction order requires that the optical path length difference is $2 \pi$ leading to $[14,15]$

$$
\Phi(\lambda)=\frac{2 \pi h_{1}}{\lambda}\left(n_{1}(\lambda)-1\right)-\frac{2 \pi h_{2}}{\lambda}\left(n_{2}(\lambda)-1\right) \stackrel{!}{=} 2 \pi .
$$

Here, the -1 in the parentheses reflects the fact that within the considered spectral range, the refractive index of air is constantly one. The above condition is equivalent to

$$
h_{1}\left(n_{1}(\lambda)-1\right)-h_{2}\left(n_{2}(\lambda)-1\right)=\lambda \text {, }
$$

which holds for any given pair of different materials and appropriate values $h_{1}, h_{2}$ at least at two different wavelengths $\lambda_{1}$ and $\lambda_{2}$, which can be chosen arbitrarily. Defining

$$
n_{i j}=n_{i}\left(\lambda_{j}\right)-1 \text { for } i, j \in\{1,2\},
$$

we obtain the two conditions

$$
\begin{aligned}
& h_{1} n_{11}-h_{2} n_{21}=\lambda_{1}, \\
& h_{1} n_{12}-h_{2} n_{22}=\lambda_{2},
\end{aligned}
$$

which can be readily solved for $h_{1}$ and $h_{2}$, if the denominator is different from zero:

$$
\begin{aligned}
& h_{1}=\frac{\lambda_{1} n_{22}-\lambda_{2} n_{21}}{n_{11} n_{22}-n_{12} n_{21}}, \\
& h_{2}=\frac{\lambda_{1} n_{12}-\lambda_{2} n_{11}}{n_{11} n_{22}-n_{12} n_{21}} .
\end{aligned}
$$

For real materials, the denominator is non-zero if the materials are different. Hence, any pair of different materials is suited to build a multilayer EA-DOE with efficiency $\eta\left(\lambda_{1}\right)=$ $\eta\left(\lambda_{2}\right)=100 \%$. However, the efficiency at other wavelengths still depends on dispersion relations of chosen materials.

Next, we show the second noteworthy characteristic that efficiency is to a certain approximation even independent of the materials. To this end let us suppose that within the wavelength range of interest, we can describe the dispersion of the refractive indices $n_{i}$ by the second order Cauchy series

$$
n_{i}(\lambda)=a_{i}+\frac{b_{i}}{\lambda^{2}}, \quad i=1,2 .
$$

Then we define

$$
n_{i j}:=a_{i}+\frac{b_{i}}{\lambda_{j}^{2}}-1, \quad i, j \in\{1,2\} .
$$

Using these relations and Eqs. (14) and (15) for the profile depths $h_{1}$ and $h_{2}$, the phase given by Eq. (9) can be written as

$\Phi(\lambda)=2 \pi x$

$$
\frac{\left(\lambda_{1} n_{22}-\lambda_{2} n_{21}\right)\left(n_{1}(\lambda)-1\right)-\left(\lambda_{1} n_{12}-\lambda_{2} n_{11}\right)\left(n_{2}(\lambda)-1\right)}{\lambda\left(n_{11} n_{22}-n_{12} n_{21}\right)} .
$$

After inserting Eqs. (16) and (17) into Eq. (18) and a somewhat lengthy, but straightforward calculation, which has been deferred to Appendix A, it turns out that $\Phi(\lambda)$ can be simplified to

$$
\begin{aligned}
\Phi(\lambda) & =2 \pi \frac{\lambda^{2} \lambda_{1}\left(\lambda_{1}+\lambda_{2}\right)+\lambda_{2}^{2}\left(\lambda^{2}-\lambda_{1}^{2}\right)}{\lambda^{3}\left(\lambda_{1}+\lambda_{2}\right)} \\
& =2 \pi \frac{\lambda^{2} \lambda_{2}\left(\lambda_{1}+\lambda_{2}\right)+\lambda_{1}^{2}\left(\lambda^{2}-\lambda_{2}^{2}\right)}{\lambda^{3}\left(\lambda_{1}+\lambda_{2}\right)} .
\end{aligned}
$$

Obviously, all material coefficients $a_{i}$ and $b_{i}$ have dropped out, which means that $\Phi(\lambda)$ is completely independent of material's refractive indices within the validity of the second order Cauchy series of Eq. (16). Again, the materials as well as the wavelengths have to be different to ensure a non-zero denominator in Eq. (18) and hence, the existence of Eq. (19). Inserting Eq. (19) in Eq. (3), an efficiency is obtained which we call generic diffraction efficiency of a multilayer EA-DOE. This term was chosen since within the approximation of Cauchy's series to the second order and under the above conditions, the generic diffraction efficiency is independent of any material parameter which is a quite remarkable result. By inspection, we see that $\Phi\left(\lambda_{1}\right)=\Phi\left(\lambda_{2}\right)=2 \pi$, which means that for the two values $\lambda_{1}$ and $\lambda_{2}$ the diffraction efficiency is indeed $100 \%$, which was to be expected since for these two wavelenghts, we required the blazing condition to hold. For other wavelengths the efficiency is, of course, less than $100 \%$, but still relatively high, as can be seen from Figure 6 .

It should be noted that even if the air gap between the two materials is filled with a third material with a different dispersion obeying a second order Cauchy relation, the result Eq. (19) would still be identical. Although the use of a third material would alter the profile depths $h_{i}$, it would, however, not improve the generic diffraction efficiencies, as one might be tempted to expect. 


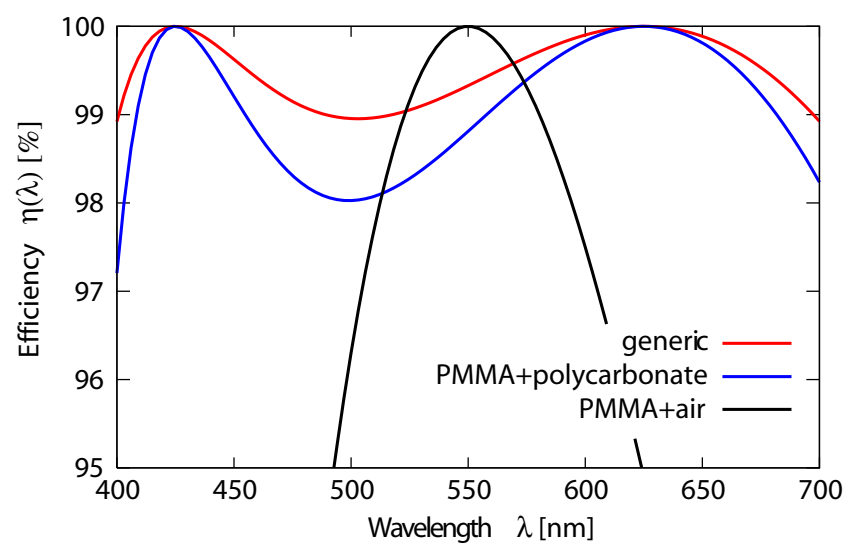

FIG. 6 Scalar diffraction efficiency of surface relief multilayer EA-DOEs with $\lambda_{1}=$ $425 \mathrm{~nm}, \lambda_{2}=625 \mathrm{~nm}$. Red: Generic diffraction efficiency calculated by Eqs. (3) and (19) under the assumption of refractive indices given by a second order Cauchy series. Blue: Diffraction efficiency of material pair polycarbonate/PMMA calculated by Eqs. (3) and (9). Black: Diffraction efficiency of the conventional DOE made from PMMA and air calculated by Eqs. (3) and (4), for comparison as already mentioned in Figure 2.

For the above derivation of a material independent efficiency, we truncated the Cauchy series after the second term. Under this assumption the first and second order of the Cauchy series can be related to the refractive index $n_{\mathrm{d}}$ and the Abbe number $v_{\mathrm{d}}$, respectively. For common depth DOEs, which do not have the air gap, the efficiency curves do depend on $n_{\mathrm{d}}$ as well as on the Abbe numbers, whereas for multilayer EA-DOEs the generic diffraction efficiency becomes materialindependent. However generally, the diffraction efficiency and also the Abbe number depend on the higher order terms of Cauchy's series Eq. (16), which can mainly be related to the relative partial dispersion $\theta_{\mathrm{g}, \mathrm{F}}$. By appropriately choosing materials with specific values of $\theta_{\mathrm{g}, \mathrm{F}}$, it is possible to further enhance the generic diffraction efficiency within the considered wavelength spectrum [22]. For a more detailed discussion of the relations between Abbe number, relative partial dispersion and Cauchy series coefficients, we refer the interested reader to Appendix A.

Now, at the end of this subsection, an example is considered for a multilayer EA-DOE made from the common plastic materials polycarbonate and PMMA. Using $\lambda_{1}=425 \mathrm{~nm}$, $\lambda_{2}=625 \mathrm{~nm}$, according to Eqs. (14) and (15), yields depths $h_{1}=16.77 \mu \mathrm{m}$ for polycarbonate and $h_{2}=13.05 \mu \mathrm{m}$ for PMMA resulting in a total depth of $h=29.82 \mu \mathrm{m}$. Figure 6 shows the diffraction efficiency for this pair of materials, which is quite similar to the generic diffraction efficiency but has a larger amplitude. This deviation is expected since the refractive indices deviate slightly from the second order Cauchy series Eq. (16).

\subsection{Sub-wavelength DOEs}

Another approach to implement blazed gratings is depicted in [23] using artificial sub-wavelength structures, which have been investigated [24] and found to be advantageous especially for imaging applications with high numerical aperture $[25,26]$. Another type of binary-blazed DOE, so-called ACES, having higher mechanical stability, was added later
[27]. Nowadays we can compare several possibilities to generate DOEs with blazing phase as given in Figure 7 where the bottom diagram shows above mentioned binary-blazed structures. The structures may consist of ridges or pillars. All parts of the structures including the air gaps must have dimensions smaller than the smallest wavelength $\lambda_{\text {short }}$ the DOE is designed for. Then, incident light of wavelengths $\lambda \geq \lambda_{\text {short }}$ cannot resolve them and therefore averages the permittivities of the ridge material and the air gap. The averaging process is wavelength dependent and accounts for a dispersion. It has been found in [6] that, using this dispersion property, by properly arranging sub-wavelength pillars and holes, EA-DOEs of only one material can be designed. In case of ridges and rings, the averaged effective refractive index is polarization dependent, and hence, some efficiency deterioration must be accepted. However, investigation of these effects is beyond the scope of this publication and are mentioned only for the sake of completeness.

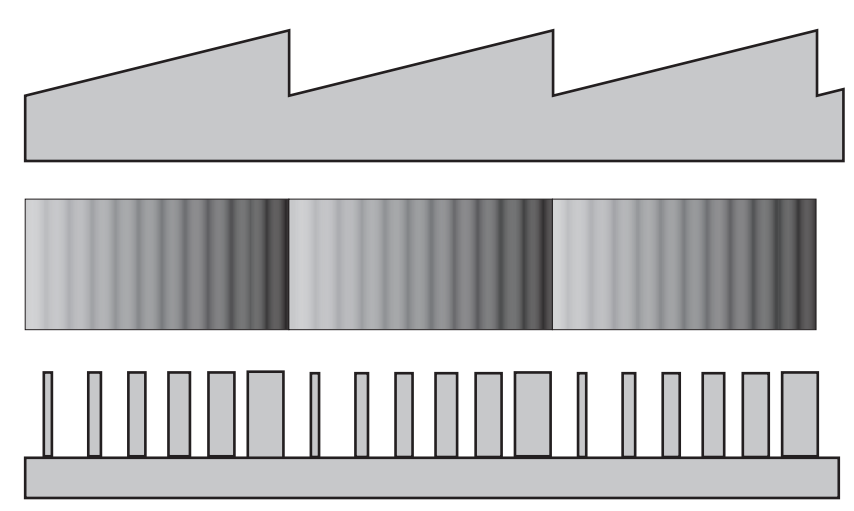

FIG. 7 Similarity between surface relief profiles (top), gradient-index materials (middle), and sub-wavelength structures (bottom) all realising a blazing phase if the effective refractive index and thickness of the DOE are chosen properly as given in Figure 8 for a GRIN-DOE.

\section{GRADIENT-INDEX EA-DOES}

\subsection{Function}

Let us start with gradient-index materials [29, 30], as a first novel design concept for EA-DOEs. Figure 7 shows the correspondence between surface relief profiles, gradient-index (GRIN) materials and sub-wavelength structures, which all serve to implement a blazing phase if the effective refractive index and the thickness $h$ of the DOE are chosen properly. Here, thickness is the proper name of quantity $h$ which corresponds to the depth of surface relief structures. The darker the grey of the GRIN-material in the middle of Figure 7 the higher is the refractive index and the larger is the optical phase delay of the light ray passing through the material at that position (cf. Figure 8). In a conventional blazing DOE with constant refractive index along the zones, the linearly increasing profile within a zone width is responsible for the blazing effect. In a GRIN element there is no profile variation, instead the refractive index has to linearly increase from the lowest value $n_{\mathrm{L}}$ at the one end of a zone $r_{\mathrm{a}}$ towards the highest value $n_{\mathrm{H}}$ at the other end of the zone $r_{\mathrm{b}}$ (cf. Figure 8). 


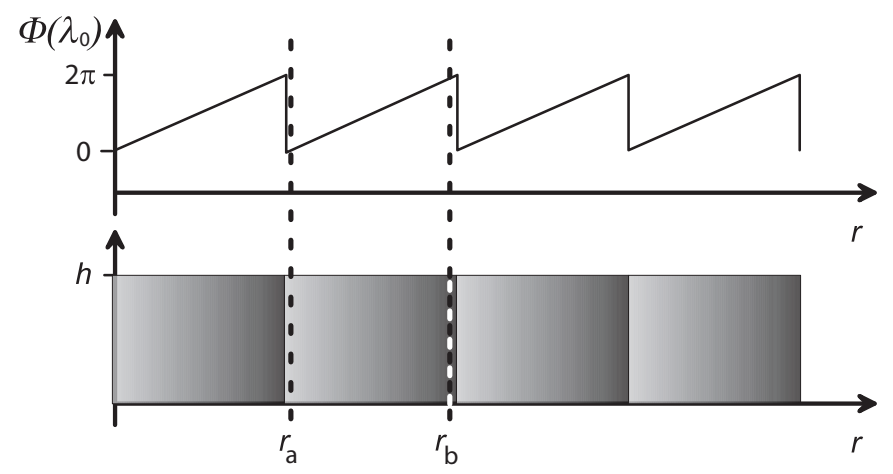

FIC. 8 Schematic representation of a GRIN-DOE (bottom) together with its phase $\Phi\left(\lambda_{0}\right)$ (top). Over a zone width between radial positions $r_{\mathrm{a}}$ and $r_{\mathrm{b}}$, a linear increasing path difference of $2 \pi$ has to be accumulated in the GRIN-material of thickness $h$. This is equivalent to a linear increase of refractive index from the minimum to the maximum value. At $r_{\mathrm{a}}$, the refractive index has a minimum while at $r_{\mathrm{b}}$, the refractive index has a maximum. The darker the grey of the GRIN-material the higher is the refractive index.

EA-DOEs using GRIN-materials consist of two separate GRIN-DOEs 1 and 2, of thicknesses $h_{1}, h_{2}$ made of different materials, but with same groove function polynomial (cf. Figure 9). It is important to align the two DOEs in a way that the position of maximum refractive index in each zone of the first material is at the position of minimum refractive index in the appropriate zone of the second material.

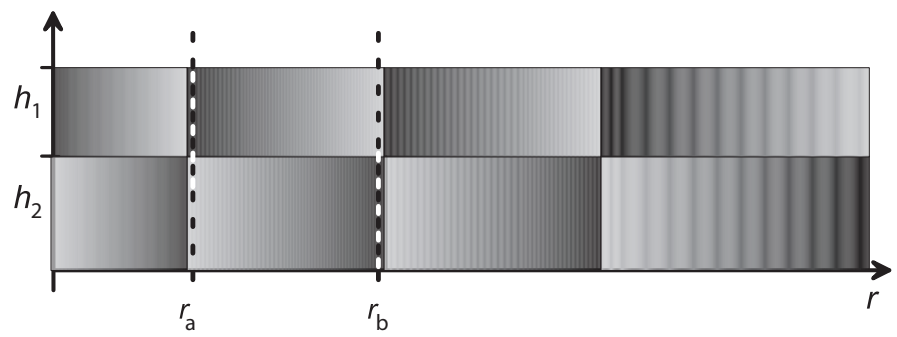

FIG. 9 Schematic representation of two layers of GRIN-DOEs from different materials to form an EA-DOE. The optical path difference is determined along paths at radial positions $r_{\mathrm{a}}$ and $r_{\mathrm{b}}$.

Now we consider two light paths over a zone width along the two lines in $r_{\mathrm{a}}$ and $r_{\mathrm{b}}$ (cf. Figure 9) with $n_{1, \mathrm{~L}}, n_{1, \mathrm{H}}$ being low and high refractive index of material 1 , respectively, and $n_{2, \mathrm{~L}}, n_{2, \mathrm{H}}$ being low and high refractive index of a different material 2, respectively. For constructive interference, the phase $\Phi(\lambda)$ being the path difference of light paths in $r_{\mathrm{a}}$ and $r_{\mathrm{b}}$ has to be $2 \pi$ :

$$
\Phi(\lambda)=\frac{2 \pi}{\lambda}\left(n_{1, \mathrm{H}} h_{1}+n_{2, \mathrm{~L}} h_{2}-n_{1, \mathrm{~L}} h_{1}-n_{2, \mathrm{H}} h_{2}\right) \stackrel{!}{=} 2 \pi,
$$

and it follows the condition

$$
\Delta n_{1}(\lambda) h_{1}-\Delta n_{2}(\lambda) h_{2}=\lambda
$$

with

$\Delta n_{1}(\lambda)=n_{1, \mathrm{H}}(\lambda)-n_{1, \mathrm{~L}}(\lambda)$ and $\Delta n_{2}(\lambda)=n_{2, \mathrm{H}}(\lambda)-n_{2, \mathrm{~L}}(\lambda)$.

Again, as known from Eqs. (9) to (15) for multilayer EADOEs, the above condition can be fulfilled for two different materials at two different wavelengths $\lambda_{1}, \lambda_{2}$ resulting in two values for thicknesses $h_{1}, h_{2}$ with scalar efficiency $\eta\left(\lambda_{1}\right)=$ $\eta\left(\lambda_{2}\right)=100 \%$ and very high efficiency over a broad spectral range, by inserting Eq. (20) into Eq. (3). The difference in materials ensures that thicknesses $h_{1}, h_{2}$ can be determined. Hence, any pair of different GRIN-materials is suited to build a GRIN EA-DOE with such an efficiency property. As the refractive index difference for GRIN-materials is smaller than for surface relief structures, the resulting thicknesses are much larger. In Table 1 two material pairs are given for ion exchange glasses from SCHOTT AG and GRINTECH AG together with the resulting thicknesses using condition in Eq. (21). The following remarks give some additional information on the choice of parameters of the considered GRIN-glasses from Table 1:

- A maximum refractive index increase of 0.1 from $n_{\mathrm{d}}=$ 1.47381 to $n_{\mathrm{d}}=1.57381$ can be achieved by a thermal ion exchange process for glass BGG 31 from SCHOTT AG with $v_{\mathrm{d}}=63.47$ which is assumed to be a constant for this GRIN-glass.

- An Ag-Na ion exchange process is applied to GRINTECH glass GT_Ag, exchanging $\mathrm{Na-ions} \mathrm{by} \mathrm{Ag-ions.}$ The refractive index can be increased by 0.14 from 1.51 to 1.65 at $\lambda_{\text {ref }}=670 \mathrm{~nm}$. Using a Li-Na ion exchange process for GRINTECH glass GT_Li, the refractive index can only be increased by 0.013 from 1.511 to 1.524 at $\lambda_{\text {ref }}$.

The dispersion of SCHOTT glass BGG 31 is fully described by the parameters $n_{\mathrm{d}}, v_{\mathrm{d}}$ given above. On the other hand, the dispersion relations of GRINTECH glasses use an expansion of Sellmeier's formula with $n_{\text {ref }}$ at $\lambda_{\text {ref }}=670 \mathrm{~nm}$ and constants $K_{11}, K_{13}$ [28] according to

$$
n^{2}(\lambda)=n^{2}\left(\lambda_{\text {ref }}\right)+K_{1}\left(n\left(\lambda_{\text {ref }}\right)\right) \cdot \frac{\left(\lambda^{2}-\lambda_{\text {ref }}^{2}\right)}{\lambda^{2}},
$$

where the function $K_{1}$ is described by the expression

$$
K_{1}\left(n\left(\lambda_{\text {ref }}\right)\right)=K_{11}+K_{13} n^{2}\left(\lambda_{\text {ref }}\right) .
$$

Constants $K_{11}, K_{13}$ are included in Table 1 for the considered two glasses.

Figure 10 shows the resulting efficiency behaviour for the first material pair of Table 1 . Obviously very high efficiency over a broad spectral range can be achieved, which is comparable to that obtained for a surface relief multilayer EA-DOE in Figure 6 at the end of Section 2.3. Clearly, the thickness of GRIN EA-DOE with about $90 \mu \mathrm{m}$ is much larger than the resulting $30 \mu \mathrm{m}$ of the surface relief example. This fact can only be improved with materials allowing a larger refractive index difference to be generated. Some new developments in this direction are cited in the next subsection. Although the second material combination in Table 1 has nearly the same efficiency behaviour, due to the small refractive index difference of the first material, the resulting thickness of more than $700 \mu \mathrm{m}$ is too large for a real application.

At the end of this subsection we show an equivalent relation as was proven for multilayer EA-DOEs in Section 2.3, 


\begin{tabular}{|c|c|c|c|c|c|}
\hline $\begin{array}{l}\text { Material } 1 \text { with } \\
\text { dispersion constants }\end{array}$ & $\lambda_{1}[\mathrm{~nm}]$ & $h_{1}[\mu \mathrm{m}]$ & $\begin{array}{l}\text { Material } 2 \text { with } \\
\text { dispersion constants }\end{array}$ & $\lambda_{2}[\mathrm{~nm}]$ & $\overline{h_{2}[\mu \mathrm{m}]}$ \\
\hline $\begin{array}{l}\text { GT_Ag }(\text { GRINTECH AG), } \\
n_{1, \mathrm{~L}}\left(\lambda_{\text {ref }}=670 \mathrm{~nm}\right)=1.51 \\
n_{1, \mathrm{H}}\left(\lambda_{\text {ref }}=670 \mathrm{~nm}\right)=1.65 \\
K_{11}=0.11098 \\
K_{13}=-0.06172\end{array}$ & 425 & 33.9 & $\begin{array}{l}\text { BGG } 31 \text { (SCHOTT AG), } \\
n_{2, \mathrm{~L}}\left(\lambda_{\mathrm{d}}\right)=1.47381, v_{\mathrm{d}}=63.47 \\
n_{2, \mathrm{H}}\left(\lambda_{\mathrm{d}}\right)=1.57381, v_{\mathrm{d}}=63.47\end{array}$ & 625 & 56.4 \\
\hline $\begin{array}{l}\text { GT_Li (GRINTECH AG), } \\
n_{1, \mathrm{~L}}\left(\lambda_{\text {ref }}=670 \mathrm{~nm}\right)=1.511 \\
n_{1, \mathrm{H}}\left(\lambda_{\text {ref }}=670 \mathrm{~nm}\right)=1.524 \\
K_{11}=0.05735 \\
K_{13}=-0.03843\end{array}$ & 425 & 669.5 & $\begin{array}{l}\text { GT_Ag (GRINTECH AG), } \\
n_{1, \mathrm{~L}}\left(\lambda_{\text {ref }}=670 \mathrm{~nm}\right)=1.51 \\
n_{1, \mathrm{H}}\left(\lambda_{\text {ref }}=670 \mathrm{~nm}\right)=1.65 \\
K_{11}=0.11098 \\
K_{13}=-0.06172\end{array}$ & 625 & 57.54 \\
\hline
\end{tabular}

TABLE 1 Two pairs of materials for GRIN EA-DOES.

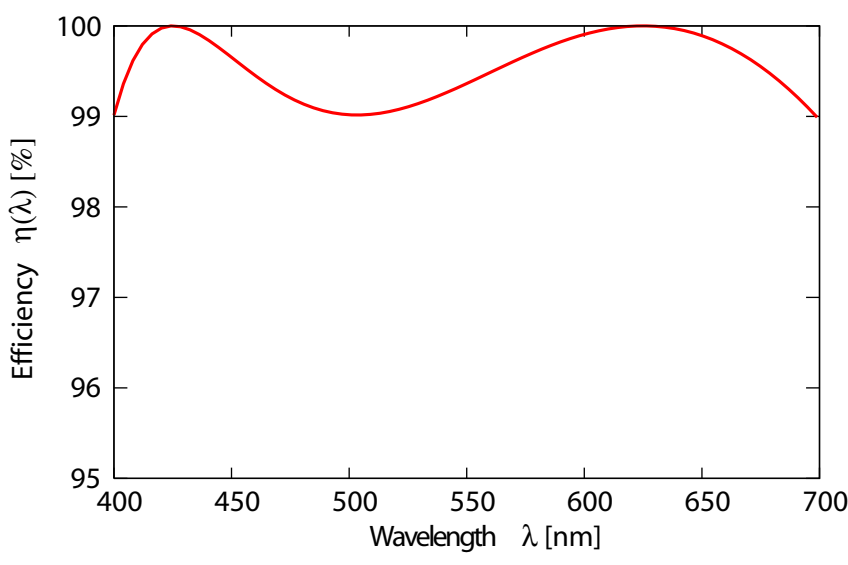

FIC. 10 Scalar diffraction efficiency of GRIN EA-DOE from materials SCHOTT BGG 31 and GRINTECH glass GT_Ag (first material pair of Table 1 calculated by Eqs. (3) and (20).

namely an efficiency which is independent of the dispersion relations if material properties can be described by second order Cauchy series:

$$
n_{i, \mathrm{H}}(\lambda)=a_{i, \mathrm{H}}+\frac{b_{i, \mathrm{H}}}{\lambda^{2}}, \quad n_{i, \mathrm{~L}}(\lambda)=a_{i, \mathrm{~L}}+\frac{b_{i, \mathrm{~L}}}{\lambda^{2}}, \quad i=1,2 .
$$

Using a similar notation as in Section 2.3 by defining

$$
\Delta n_{i j}:=n_{i, \mathrm{H}}\left(\lambda_{j}\right)-n_{i, \mathrm{~L}}\left(\lambda_{j}\right) \text { for } i, j \in\{1,2\} \quad,
$$

according to Eq. (22), we obtain the two following expressions

$$
\begin{aligned}
& h_{1} \Delta n_{11}-h_{2} \Delta n_{21}=\lambda_{1}, \\
& h_{1} \Delta n_{12}-h_{2} \Delta n_{22}=\lambda_{2},
\end{aligned}
$$

for two arbitrary but different wavelengths $\lambda_{1}, \lambda_{2}$. The equations can be readily solved for $h_{1}$ and $h_{2}$, if the materials are different. Inserting the results for $h_{1}, h_{2}$ and the Cauchy relations Eq. (25) into Eq. (20), after some lengthy simplifications, it yields the result being identical the same expression Eq. (19) as for the multilayer EA-DOE. Again, all material coefficients $a_{i}$ and $b_{i}$ have dropped out, which means that $\Phi(\lambda)$ is completely independent of material's refractive indices within the validity of the second order Cauchy series. Therefore, also the generic GRIN efficiency is identical to the generic efficiency of the multilayer EA-DOE. So, we may refer to Figure 6 for an example of this really generic efficiency behaviour.

\subsection{Fabrication}

For fabrication of GRIN EA-DOEs we are only able to give some rough hints. GRIN glasses given in Table 1 use thermal ion exchange processes for refractive index modifications, in some cases assisted by electric fields [29, 30]. Other materials suitable to generate GRIN-DOEs are e.g. photopolymers and chalcogenide glasses [31]. For writing a GRIN-profile into chalcogenide glass, a wavelength must be used which is below the critical wavelength where the glass is photosensitive. After writing, the glass behaves like a conventional GRINglass for wavelengths above the critical wavelength. Corresponding writing techniques are e.g. holography, grey-tone mask exposure, and direct laser writing. In a similar way also in photopolymers refractive index profiles can be generated. Such techniques and applications of photopolymers can e.g. be found in [32]-[35]. In [32] a novel photopolymer is proposed, in which a refractive index difference up to $\Delta n=0.45$ has been generated at $\lambda=633 \mathrm{~nm}$. Another novel photopolymer with refractive index difference up to $\Delta n>0.5$ was proposed in [33], where wavelengths $\lambda=633 \mathrm{~nm}$ and $\lambda=670 \mathrm{~nm}$ were used. These values exceed by far the refractive index differences of $\Delta n=0.1 \ldots 0.14$ in the examples of Table 1. However, these large refractive index differences proposed in $[32,33]$, were possible to fabricate only by generating a polarization dependent birefringence in the material. Hence, these materials cannot directly be used in our concepts. Nevertheless, materials having a similar large refractive index differences without birefringence would lead to significant smaller thicknesses, of e.g. one third of the values given in Table 1 which is equal to about $30 \mu \mathrm{m}$. This value is already in the magnitude of the thickness for surface relief EA-DOEs.

\section{SUB-WAVELENGTH EA-DOES}

\section{1 Function}

Within this section we now consider sub-wavelength structures to create EA-DOEs. The correspondence between surface relief profiles, gradient-index materials, and subwavelengths structures (cf. Figure 7) allows us to do this. EA-DOEs using sub- $\lambda$-structures consist of a single layer made from two media: 1 and 2. This layer can be on top 
of any transparent substrate. $v_{1}(\vec{r})$ and $v_{2}(\vec{r})$ designate the volume fractions of the first and second medium, respectively, at position $\vec{r}$ in a cube with volume well below $\lambda_{\text {short }}^{3}$. As will be seen later, their refractive indices $n_{1}(\lambda)$ and $n_{2}(\lambda)$ have to be related to each other. The EA-DOE is assumed to work in the wavelength band between $\lambda_{\text {short }}$ and $\lambda_{\text {long }}$.

Since light of wavelength $\lambda_{\text {short }} \leq \lambda \leq \lambda_{\text {long }}$ only resolves structures with dimensions larger than $\lambda$, the interaction of light with sub- $\lambda$-structures smaller than $\lambda_{\text {short }}$ can be described by effective medium theory (EMT) [36]. As already mentioned in Section 2.4, light averages the permittivities $\epsilon_{1}$ and $\epsilon_{2}$ of the two materials. These relations are polarization dependent and we have to consider that $n^{2}=\epsilon$. Then, for TE-polarization, the nonlinear relation

$$
\begin{aligned}
n_{\mathrm{eff}}(\lambda, \vec{r})= & \sqrt{v_{1}(\vec{r}) n_{1}^{2}(\lambda)+v_{2}(\vec{r}) n_{2}^{2}(\lambda)} \\
& \text { with } \quad v_{1}(\vec{r})+v_{2}(\vec{r})=1
\end{aligned}
$$

holds for the refractive index of an effective medium. On the other hand, for TM-polarization, the effective refractive index can be calculated by the nonlinear relation

$$
n_{\mathrm{eff}}(\lambda, \vec{r})=\frac{1}{\sqrt{\frac{v_{1}(\vec{r})}{n_{1}^{2}(\lambda)}+\frac{v_{2}(\vec{r})}{n_{2}^{2}(\lambda)}}} \quad \text { with } \quad v_{1}(\vec{r})+v_{2}(\vec{r})=1 .
$$

A small calculation given in Appendix B shows that both functional expressions Eq. (29), Eq. (30) can be approximated with sufficient accuracy by the the same linear relation

$$
\begin{aligned}
n_{\text {eff }}(\lambda, \vec{r})= & v_{1}(\vec{r}) n_{1}(\lambda)+v_{2}(\vec{r}) n_{2}(\lambda) \\
& \text { with } \quad v_{1}(\vec{r})+v_{2}(\vec{r})=1 .
\end{aligned}
$$

In the following and for simplicity, we restrict ourselves to rotationally symmetrical functions of $v_{1}(\vec{r})$ and $v_{2}(\vec{r})$, i. e. $v_{1}(\vec{r})=v_{1}(r)$ and $v_{2}(\vec{r})=v_{2}(r)$ where $r=|\vec{r}|$ is the distance from the optical axis. This simplifies the notation and it is the most typical application in connection with lenses. Moreover, the utilisation for linear gratings is straightforward.

By proper choice of volume fraction $v_{1}(r)$, such a layer forms clearly a DOE. As an example, imagine a rotationally summetrical layer where the volume fraction $v_{1}(r)$ varies like a sawtooth as shown in Figure 11. Thickness $h$ of the layer is chosen according to

$$
\begin{aligned}
h & \equiv \frac{\lambda_{0}}{n_{\mathrm{eff}}\left(\lambda_{0}, r_{\mathrm{b}}\right)-n_{\mathrm{eff}}\left(\lambda_{0}, r_{\mathrm{a}}\right)} \\
& =\frac{\lambda_{0}}{\left(v_{1}\left(r_{\mathrm{b}}\right)-v_{1}\left(r_{\mathrm{a}}\right)\right)\left(n_{1}\left(\lambda_{0}\right)-n_{2}\left(\lambda_{0}\right)\right)}
\end{aligned}
$$

where again, points with radial distance $r_{\mathrm{a}}$ and $r_{\mathrm{b}}$ are located near both sides of the discontinuity of $v_{1}(r)$. The distance between adjacent discontinuities of $v_{1}(r)$ corresponds to the zone width $d$. Light passing through this layer undergoes a periodic phase shift which is discontinuous at discontinuities of $v_{1}(r)$; the discontinuity in phase shift between positions $r_{\mathrm{a}}$ and $r_{\mathrm{b}}$ is given by

$$
\begin{aligned}
\Phi(\lambda) & =\frac{2 \pi}{\lambda}\left(n_{\mathrm{eff}}\left(\lambda, r_{\mathrm{b}}\right) h-n_{\mathrm{eff}}\left(\lambda, r_{\mathrm{a}}\right) h\right) \\
& =\frac{2 \pi \lambda_{0}}{\lambda} \frac{n_{\mathrm{eff}}\left(\lambda, r_{\mathrm{b}}\right)-n_{\mathrm{eff}}\left(\lambda, r_{\mathrm{a}}\right)}{n_{\mathrm{eff}}\left(\lambda_{0}, r_{\mathrm{b}}\right)-n_{\mathrm{eff}}\left(\lambda_{0}, r_{\mathrm{a}}\right)} .
\end{aligned}
$$

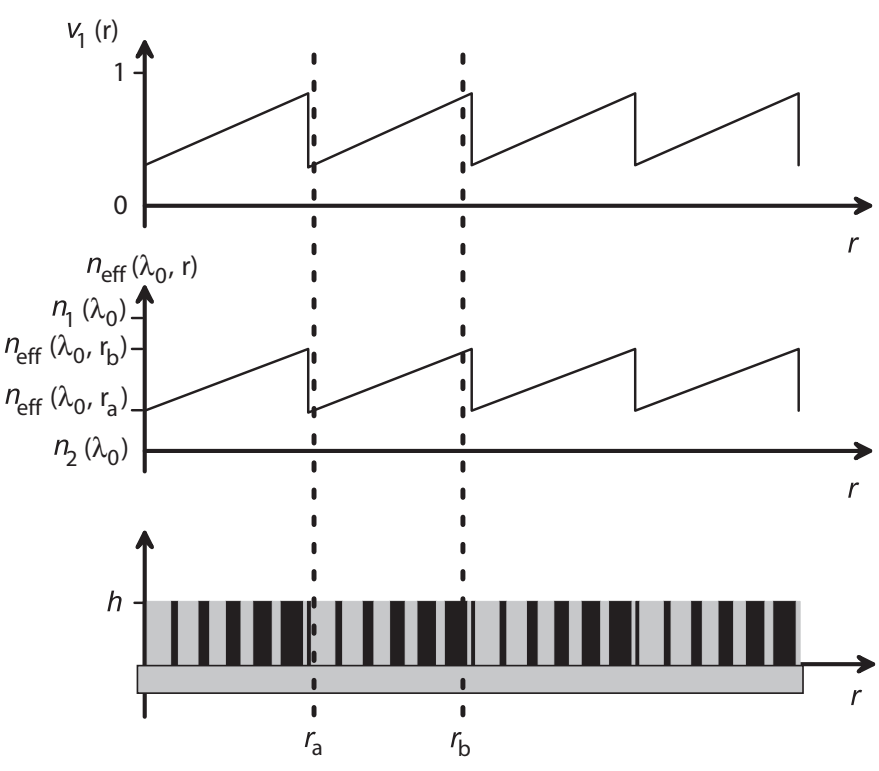

FIG. 11 Visualization of Eq. (31) exemplified for a sub-wavelength EA-DOE with constant zone width $d=\left|r_{\mathrm{a}}-r_{\mathrm{b}}\right|$. Upper diagram: Volume fraction $v_{1}(r)$ of the first material as a function of distance $r$ from the center of the DOE. Middle: Effective refractive index $n_{\text {eff }}\left(\lambda_{0}, r\right)$ at design wavelength $\lambda_{0}$ as a function of $r$. Lower diagram: Cross section of a binary sub-wavelength EA-DOE layer with thickness $h$ and its two planar surfaces. Black and light grey correspond to the two materials the EA-DOE consists of, generating an effective index of refraction between $n_{\text {eff }}\left(\lambda_{0}, r_{\mathrm{a}}\right)$ and $n_{\text {eff }}\left(\lambda_{0}, r_{\mathrm{b}}\right)$ respectively where $r_{\mathrm{a}}$ and $r_{\mathrm{b}}$ denote positions located near both sides of the discontinuities of $v_{1}(r)$.

Obviously, it follows $\Phi\left(\lambda_{0}\right)=2 \pi$; i. e. light with design wavelength $\lambda_{0}$ is diffracted to the first order with a scalar diffraction efficiency of $\eta\left(\lambda_{0}\right)=100 \%$. However, this DOE is not yet an EA-DOE since $\Phi(\lambda)$ still depends on $\lambda$.

To ensure that above layer is a truly EA-DOE with scalar diffraction efficiency $\eta(\lambda)=100 \%$ for all wavelengths $\lambda$ between $\lambda_{\text {short }}$ and $\lambda_{\text {long, }}$ the refractive indices $n_{1}(\lambda)$ and $n_{2}(\lambda)$ of media 1 and 2 must be related. As will be shown, from the relation

$$
\begin{aligned}
n_{1}(\lambda)= & n_{2}(\lambda)+\frac{\lambda}{\lambda_{0}}\left(n_{1}\left(\lambda_{0}\right)-n_{2}\left(\lambda_{0}\right)\right) \\
& \text { for } \quad \lambda_{\text {short }} \leq \lambda \leq \lambda_{\text {long }}
\end{aligned}
$$

which is the same as Eq. (8), it follows that $\Phi(\lambda)=2 \pi$ and $\eta(\lambda)=100 \%$ in scalar approximation for the wavelength band under consideration. This can be proven as follows. From Eqs. (31) and (34) one obtains

$$
\begin{aligned}
& n_{\mathrm{eff}}(\lambda, r)=v_{1}(r) n_{1}(\lambda)+v_{2}(r) n_{2}(\lambda) \\
& \quad=v_{1}(r)\left[n_{2}(\lambda)+\frac{\lambda}{\lambda_{0}}\left(n_{1}\left(\lambda_{0}\right)-n_{2}\left(\lambda_{0}\right)\right)\right]+v_{2}(r) n_{2}(\lambda) \\
& =\left(v_{1}(r)+v_{2}(r)\right) n_{2}(\lambda)+v_{1}(r) \frac{\lambda}{\lambda_{0}}\left(n_{1}\left(\lambda_{0}\right)-n_{2}\left(\lambda_{0}\right)\right) .
\end{aligned}
$$

Abbreviation $\Delta n\left(\lambda_{0}\right) \equiv n_{1}\left(\lambda_{0}\right)-n_{2}\left(\lambda_{0}\right)$ together with relation $v_{1}(r)+v_{2}(r)=1$ leads to

$$
n_{\mathrm{eff}}(\lambda, r)=n_{2}(\lambda)+v_{1}(r) \frac{\lambda}{\lambda_{0}} \Delta n\left(\lambda_{0}\right) .
$$


Thus, we may write

$$
\begin{aligned}
& n_{\text {eff }}\left(\lambda, r_{\mathrm{a}}\right)-n_{\text {eff }}\left(\lambda, r_{\mathrm{b}}\right)= \\
& n_{2}(\lambda)+v_{1}\left(r_{\mathrm{a}}\right) \frac{\lambda}{\lambda_{0}} \Delta n\left(\lambda_{0}\right)-n_{2}(\lambda)-v_{1}\left(r_{\mathrm{b}}\right) \frac{\lambda}{\lambda_{0}} \Delta n\left(\lambda_{0}\right)= \\
& \frac{\lambda}{\lambda_{0}} \Delta n\left(\lambda_{0}\right)\left(v_{1}\left(r_{\mathrm{a}}\right)-v_{1}\left(r_{\mathrm{b}}\right)\right) .
\end{aligned}
$$

From Eq. (33), the discontinuity of the phase shift obeys

$$
\begin{aligned}
\Phi(\lambda) & =\frac{2 \pi \lambda_{0}}{\lambda} \frac{n_{\mathrm{eff}}\left(\lambda, r_{\mathrm{b}}\right)-n_{\mathrm{eff}}\left(\lambda, r_{\mathrm{a}}\right)}{n_{\mathrm{eff}}\left(\lambda_{0}, r_{\mathrm{b}}\right)-n_{\mathrm{eff}}\left(\lambda_{0}, r_{\mathrm{a}}\right)} \\
& =\frac{2 \pi \lambda_{0}}{\lambda} \frac{\frac{\lambda}{\lambda_{0}} \Delta n\left(\lambda_{0}\right)\left(v_{1}\left(r_{\mathrm{b}}\right)-v_{1}\left(r_{\mathrm{a}}\right)\right)}{\frac{\lambda_{0}}{\lambda_{0}} \Delta n\left(\lambda_{0}\right)\left(v_{1}\left(r_{\mathrm{b}}\right)-v_{1}\left(r_{\mathrm{a}}\right)\right)} \\
& =\frac{2 \pi \lambda_{0}}{\lambda} \frac{\lambda}{\lambda_{0}} \\
& =2 \pi .
\end{aligned}
$$

Thus, $\Phi(\lambda)$ is actually a constant and the proposed sub- $\lambda-$ structure really forms an EA-DOE in the wavelengths band between $\lambda_{\text {short }}$ and $\lambda_{\text {long. Remarkably, }} \Phi(\lambda)$ does not depend on the volume difference $v_{1}\left(r_{\mathrm{b}}\right)-v_{1}\left(r_{\mathrm{a}}\right)$. It is possible to specify $v_{1}\left(r_{\mathrm{a}}\right), v_{1}\left(r_{\mathrm{b}}\right)$ according to e.g. fabrication needs, which determines the thickness $h$ but leaves the efficiency unchanged. For fabrication reasons it can be advantageous to choose a volume difference $v_{1}\left(r_{\mathrm{b}}\right)-v_{1}\left(r_{\mathrm{a}}\right)$ smaller than 1 . Clearly, because of Eq. (32), then thickness $h$ becomes larger as if in the case $v_{1}\left(r_{\mathrm{b}}\right)-v_{1}\left(r_{\mathrm{a}}\right)=1$.

In reality, one hardly ever finds two media which fulfill Eq. (34) exactly. However, there are many pairs of materials with refractive indices fulfilling Eq. (34) approximately. Since refractive index Eqs. (8) and (34) are identical, material pairs suited for common depth EA-DOEs are also appropriate for sub- $\lambda$-structure EA-DOEs. Using Eqs. (31), (33) and inserting it into Eq. (3), it can be shown that the diffraction efficiency of above sub- $\lambda$-structure is given by the phase function Eq. (6) together with Eq. (3), which is the same as resulted for surface relief EA-DOEs with common depth. This is not really surprising since already condition in Eq. (34) is the same as Eq. (8) for surface relief EA-DOEs.

\subsection{Pairs of materials for sub- $\lambda$-structure EA-DOES}

In this subsection, examples for pairs of materials forming sub- $\lambda$-structure EA-DOEs are given. Their diffraction efficiency in the visual band between $\lambda_{\text {short }}=400 \mathrm{~nm}$ and $\lambda_{\text {long }}=700 \mathrm{~nm}$ exceeds by far the diffraction efficiency of a conventional DOE consisting of PMMA and air shown in Figure 6.

In our examples, we always combine a glass with a plastic medium. Such material pairs are e.g. N-BaF52 (SCHOTT AG) and polycarbonate, K-LaFK60 (Sumita) and polycarbonate, and K-VC81 (Sumita) and Ultem (GE Plastics). The refractice indices for polycarbonate and optical glass can be taken from any commercial optical design software. The refractive indices of Ultem are calculated according to

$$
n_{\text {Ultem }}(\lambda)=1.6034+18828 \mathrm{~nm}^{2} \lambda^{-2} .
$$

Using this expression, refractive indices found in the internet [37] can be reproduced within good approximation. Figure 12 summarizes dispersion curves for the three pairs of materials given above.
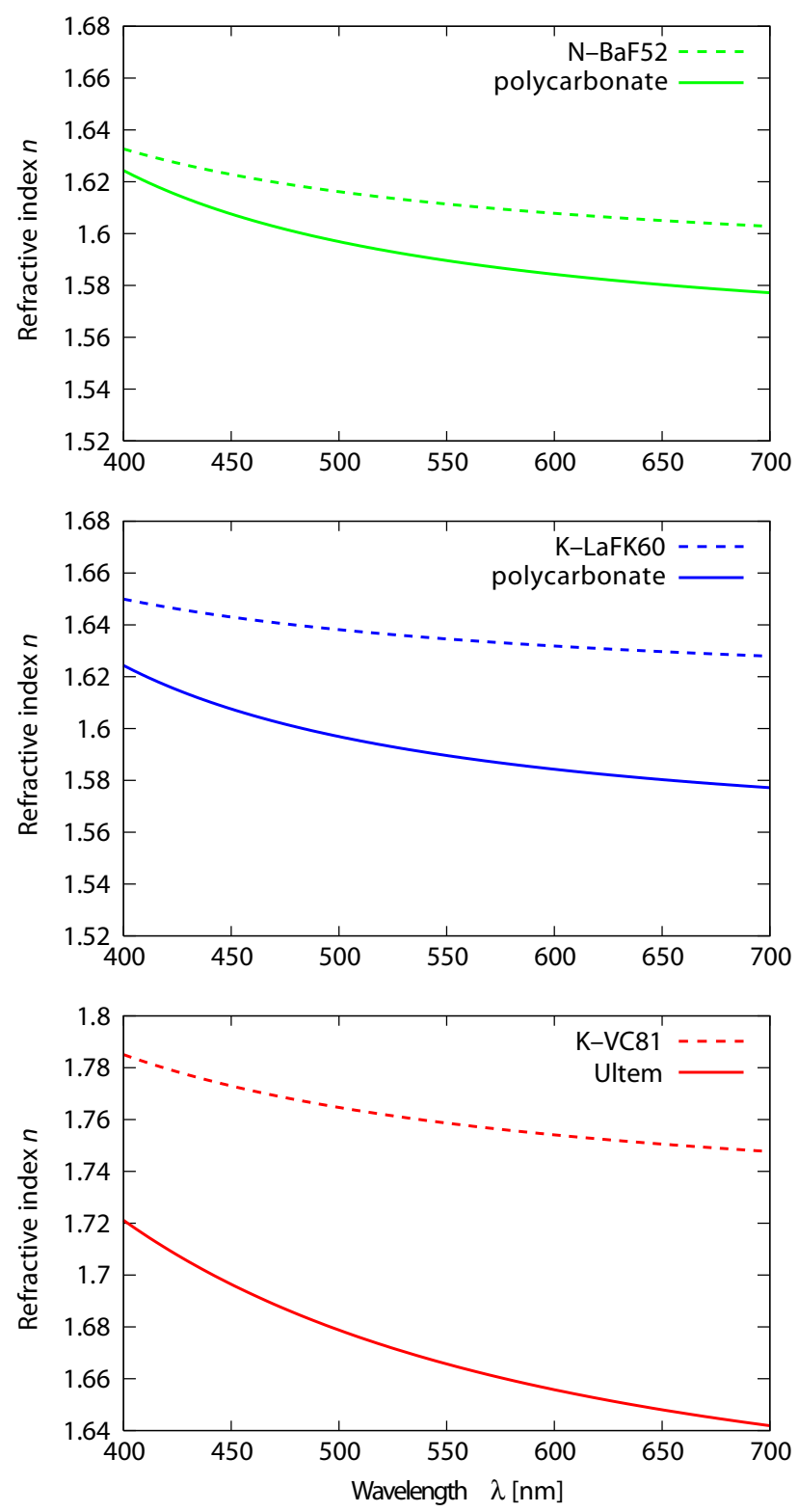

FIG. 12 Refractive index curves $n(\lambda)$ corresponding to dispersion relations for material pairs $\mathrm{N}-\mathrm{BaF}_{52}$ and polycarbonate (upper diagram), K-LaFK60 and polycarbonate (middle), K-VC81 and Ultem (lower diagram).

Finally, diffraction efficiencies for these pairs of materials according to the phase function Eq. (6) together with Eq. (3) are given in Figure 13. The design wavelength $\lambda_{0}$ has been chosen to be $\lambda_{0}=500 \mathrm{~nm}$ for the pair N-BaF52 and polycarbonate, $\lambda_{0}=575 \mathrm{~nm}$ for the pair K-LaFK60 and polycarbonate and $\lambda_{0}=560 \mathrm{~nm}$ for pair K-VC81 and Ultem. Additionaly, Figure 13 shows the diffraction efficiency of a conventional DOE for materials PMMA and air in scalar approximation. This figure clearly demonstrates that the diffraction efficiency of the proposed sub- $\lambda$-structure EA-DOE exceeds the corresponding value of a conventional DOE. 


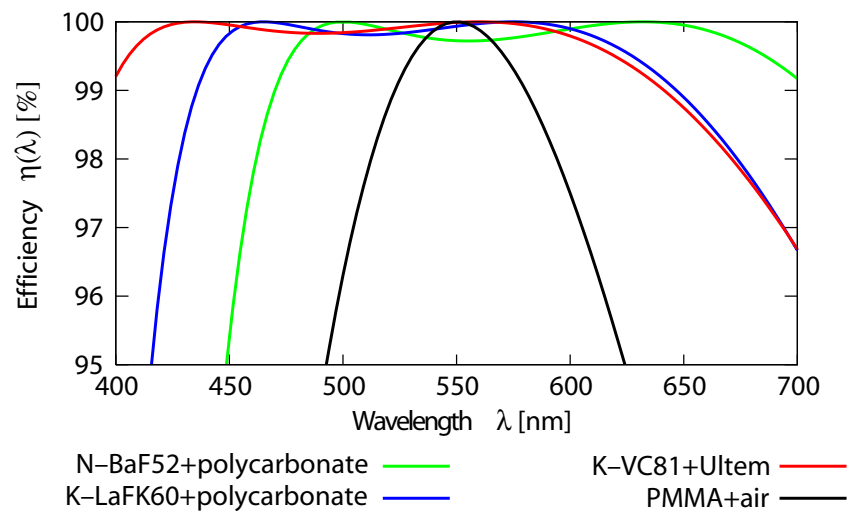

FIG. 13 Scalar diffraction efficiency of EA-DOEs according to Eqs. (3) and (6) for three pairs of materials. These curves hold for common depth EA-DOEs as well as for sub- $\lambda$ structure EA-DOEs. For comparison, the scalar diffraction efficiency of a conventional surface relief DOE made from PMMA and air is also shown.

\subsection{Thickness $h$ of sub- $\lambda$-structure EA-DOES}

According to Eq. (32), thickness $h$ of the proposed sub- $\lambda-$ structure EA-DOE depends on chosen materials as well as on the volume fraction $v_{1}(r)$. Figure 14 shows this dependency for three pairs of materials given in the preceding subsection. Thickness $h$ decreases with increasing values of $v_{1}\left(r_{\mathrm{b}}\right)-v_{1}\left(r_{\mathrm{a}}\right)$ because in this case also the denominator of Eq. (32) increases.

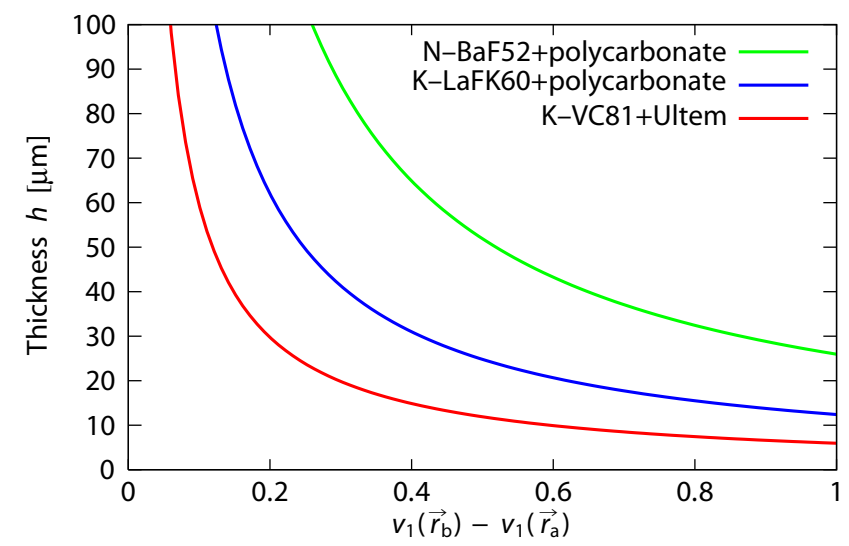

FIG. 14 Thickness $h$ of the sub- $\lambda$-structure EA-DOE according to Eq. (32) for three pairs of materials as a function of $v_{1}\left(r_{\mathrm{b}}\right)-v_{1}\left(r_{\mathrm{a}}\right)$.

Typically, thickness $h$ is smaller than $100 \mu \mathrm{m}$. However, a thinner sub- $\lambda$-structure EA-DOE is advantageous since with decreasing $h$ the diffraction efficiency depends less on the angle of incidence of the light passing through the DOE. Additionally, the diffraction efficiency due to rigorous electromagnetic effects decreases with increasing thickness $h$. Therefore, material pairs and volume fractions $v_{1}(r)$ are advantageous which lead to a thickness $h$ which is as small as possible (e.g. $h<10 \mu \mathrm{m} . .20 \mu \mathrm{m})$.

\subsection{Embodiments}

Figure 15 shows a sub- $\lambda$-structure EA-DOE which is build up from concentric alternating rings of materials 1 and 2 with refractive indices $n_{1}(\lambda)$ and $n_{2}(\lambda)$. Each zone width $d$ is composed of many of these rings. In order to form a sub- $\lambda-$ structure, it is necessary that the rings have widths well below $\lambda_{\text {short }}$. Within a zone width $d$, the width of the rings differs in order to implement the desired distribution of the volume fractions $v_{1}(r)$ and $v_{2}(r)$. Since in azimuthal direction the rings are of thickness $h$ and not sub- $\lambda$-structures, this DOE will cause some polarization effects. This structure could e.g. be manufactured by microlithography or Nano-Imprint technology [38]; the second material could also be a liquid such as an immersion oil with appropriate refractive index [39].

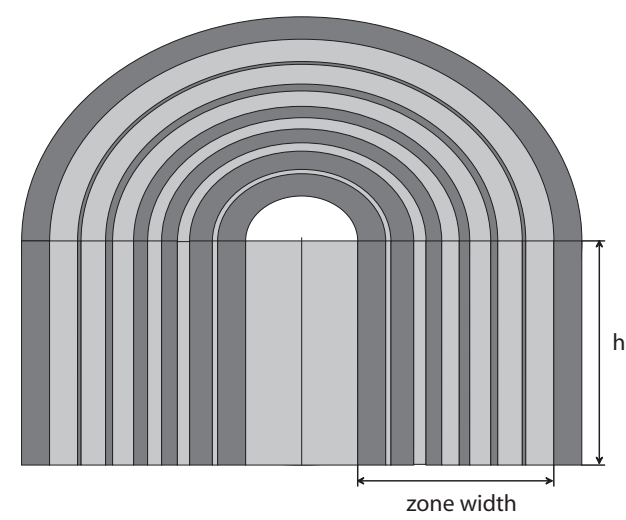

FIG. 15 Combined vertical and horizontal cross-section of sub- $\lambda$-structure EA-DOEs with concentric rings. Dark and light grey denote the first and second material. Within a local zone width $d$, there are several concentric rings with radial dimensions well below $\lambda_{\text {short }}$

Another type of sub- $\lambda$-structure with pillars made from a first material in a matrix of a second material is depicted in Figure 16. The diameter of the pillars as well as the distance between two pillars must fall well below $\lambda_{\text {short }}$ and must differ over $r$ to form the desired volume fractions $v_{1}(r)$ and $v_{2}(r)$. The pillars can be arranged in a regular or stochastic fashion. The cross sectional area of the pillars may be of arbitrary shape, e.g. circular, triangular or rectangular. In contrast to the preceding embodiment, this EA-DOE has smaller polarization effects.

A third type of sub- $\lambda$-structure is build up from pellets of a first material in a matrix of a second material, which is shown in Figure 17. Both, the diameter and the distance of the pellets are much smaller than $\lambda_{\text {short }}$. The pellets can be distributed in a regular or statistical manner and the size of the pellets may differ. Again, the given form of the volume fractions must be met. The pellets may be spherules but can also be of different shape. This type of EA-DOE does not exhibit any polarization effect.

\section{CUT-AND-PASTE EA-DOES}

Another theoretical type of EA-DOE is the so-called cut-andpaste design. It should be noted that now the dimensions exceed $\lambda_{\text {short }}$ by far; thus, it is not a sub- $\lambda$-structure. This ap- 


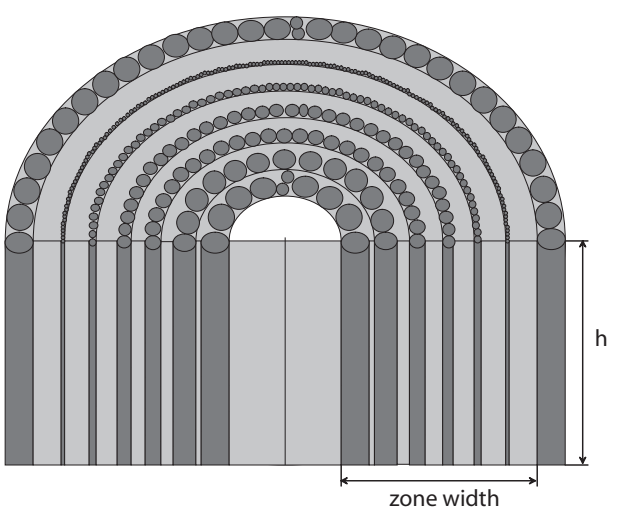

FIG. 16 Combined vertical and horizontal cross-section of sub- $\lambda$-structure EA-DOES with pillars. Dark and light grey denote the first and second material. Within a local zone width $d$, there are plenty of pillars with diameter and distance well below $\lambda_{\text {short }}$

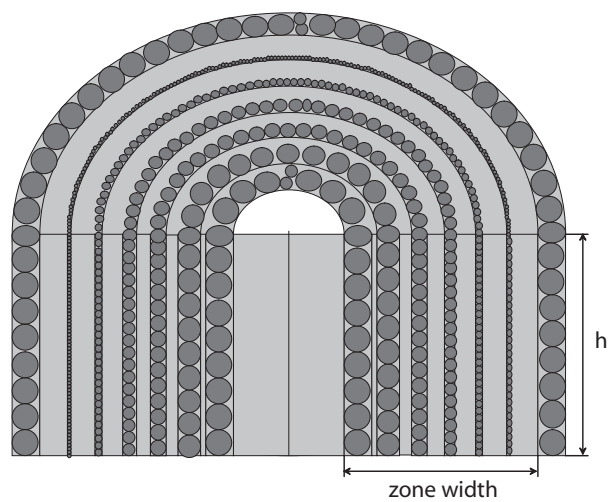

FIC. 17 Combined vertical and horizontal cross-section of sub- $\lambda$-structure EA-DOEs with pellets. Dark and light grey denote the first and second material. Within a local zone width $d$, there are a huge amount of pellets with typical dimension and distance well below $\lambda_{\text {short }}$.

proach uses the fact that the optical path length of a light ray traversing a sequence of different materials is independent of the order of this sequence, in which the different materials are arranged. Here it is used to modify the structure of the DOE-profiles by cutting a part of material at one position and pasting it at another position resulting in several possibilities of new arrangements of the two materials, which are possibly better suited to some modern manufacturing techniques. Accepting only vertical shifts and no horizontal ones of the cutted material parts, fulfils the above condition. Therefore, it is possible, to arbitrarily cut out parts of the blaze profiles of multilayer EA-DOEs and put them at different places, as long as at each position within the zone width, the optical path length remains the same. Hence, by rearranging the material composition, it is possible to create grating shapes which do not look at all like blazed gratings, nevertheless, they are fully equivalent to the original multilayer EA-DOE.

The simplest rearrangements have already been considered in Figure 5, where it is clear that the optical effects of each grating version are fully equivalent, since the optical path lengths at each lateral position within one zone width are identical. The difference between the left and the middle embodiment is only a constant offset in the thickness of the air gap, which, at least in the scalar approximation, has no impact on the optical performance. Between the middle and the right embodiment, only the sequence of materials has been altered, hence light impinging from above first sees the air and then the two materials, instead of one material first, the air gap, and then the other material.

In principle there are infinitely many possibilities to rearrange the material composition within the modulated region, however, most of them will not possess any advantage from a manufacturability point of view and therefore will be only of academic interest. A collection of various decompositions is shown in Figure 18. A more interesting structuring from a practical point of view may be the next approach [40].
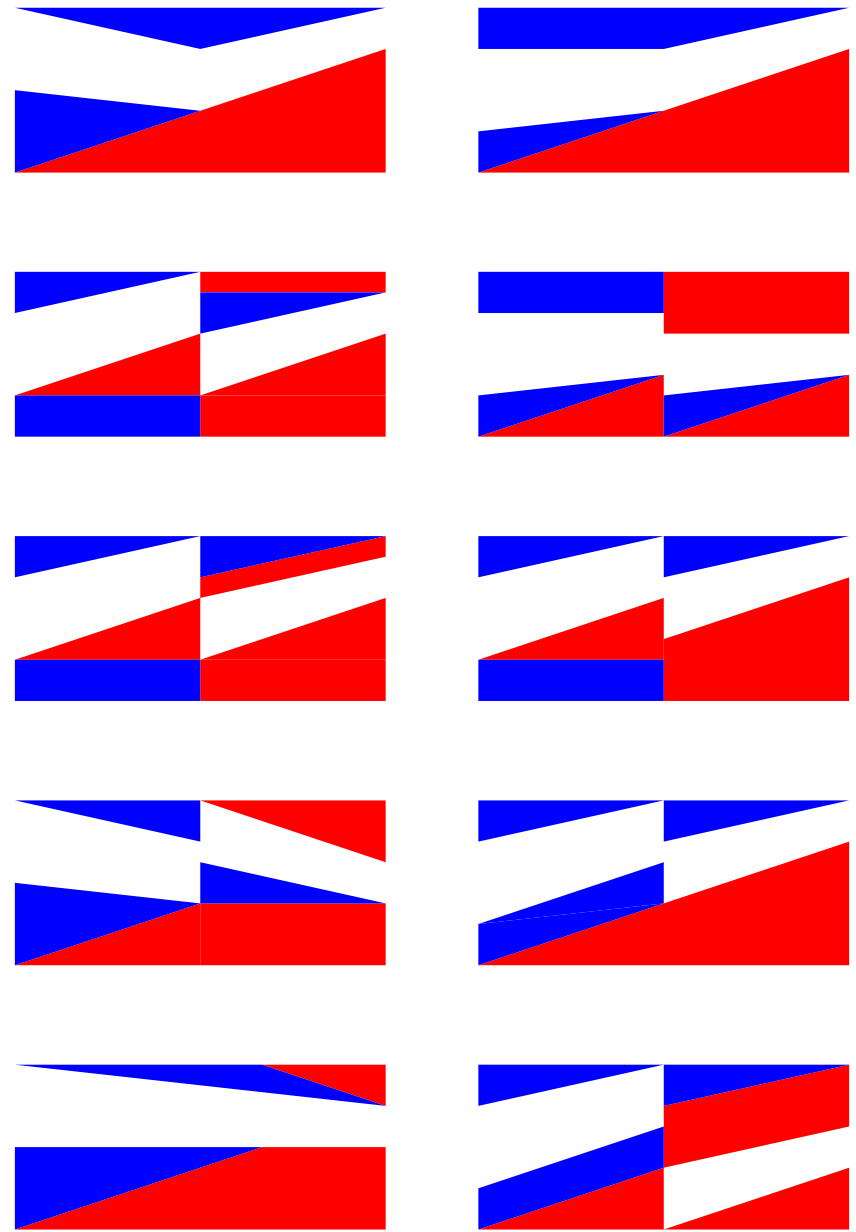

FIG. 18 A collection of several equivalent decompositions of the two materials to form another EA-DOE.

The rearrangements made before with blaze profiles can likewise be done with multilevel blaze profiles. Two equivalent rearrangements of a multilayer EA-DOE consisting of two mulitilevel blaze profiles are shown in Figure 19. The rearrangements between the red and blue parts are similar to those, known from the falling-blocks puzzle video game called Tetris [41], one of the most popular video games ever. Thus, this special cut-and-paste design has also been called Tetris-design. However, there are two main differences to the Tetris game rules: 1) the blocks are not limited to four squares, and 2) horizontal shifting of the falling-blocks is prohibited. This leaves the optical path length unchanged and ensures 
working of the designed DOE as an efficiency achromatized one.
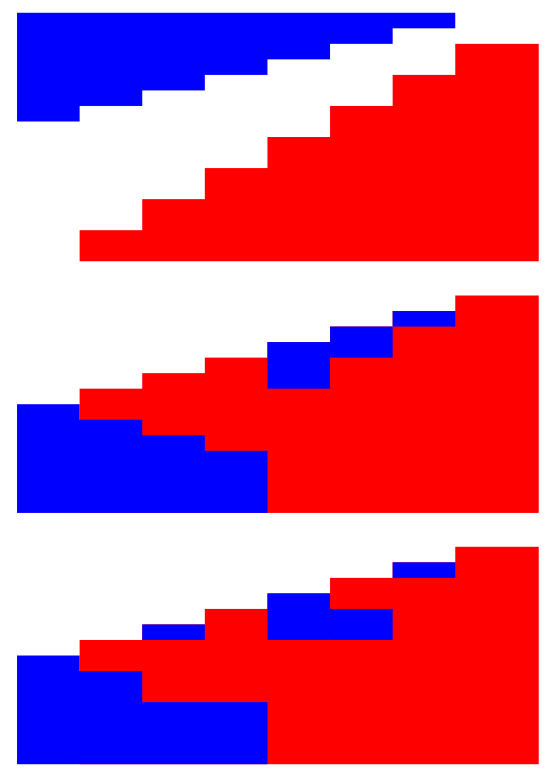

FIC. 19 Multilayer EA-DOE consisting of two mulitilevel blaze profiles (top) and two equivalent rearrangements of some material parts (middle and bottom). Corresponds to the so-called Tetris-design.

Finally it should be noted that in the scalar limit, all the shown embodiments should exactly exhibit the same diffraction efficiency behavior as a function of wavelength. In reality, they will show some difference due to different electromagnetic effects at the various boundaries, which might be the stronger, the smaller the zone width is. The influence of depth variations on the diffraction efficiency for the left and right arrangements of Figure 5 was already studied in [14, 15], where it was shown that the right arrangement was less sensitive to depth errors.

\section{DISCUSSION}

Hybrid lenses with DOEs are often utilized in lightweight optical systems for the correction of longitudinal axial chromatic aberrations as well as of the chromatic difference of magnification. Due to their excellent chromatic correction capability, EA-DOEs are well suited to correct large errors occuring in e.g. broadband optical systems without loss of efficiency. This has been recognized and applied in photographic lenses with embedded multilayer EA-DOE which are commercially available underlining the importance of this concept.

We have presented known and new concepts to design EADOEs which possess diffraction efficiency larger than $97 \%$ over a broad spectral interval, exemplified for the visible range from $400 \mathrm{~nm}$ to $700 \mathrm{~nm}$ wavelength. Successively, we discussed the application of surface relief profiles, gradientindex materials, sub-wavelength structures, and a so-called cut-and-paste design. The main idea behind all approaches (except the one in Section 2.4) to achieve the goal of effi- ciency achromatization, is to use at least two different materials which obey certain dispersion relations. From these relations, design parameters as profile heights are derived and the resulting diffraction efficiency can be deduced. For the derivation of all these relatively simple expressions we calculated the phase between two paths near the boundaries of a zone. Hence, all our approaches are based on scalar assumptions. Nevertheless, these are sufficiently fulfilled with zone widths larger than $50 \ldots 100$ times the wavelength in the case of main application, namely for correction of chromatic aberrations.

For common depth EA-DOEs the two materials have to satisfy a linear relationship in the difference of their refractive indices Eq. (8), which reduces the number of possible material combinations. The multilayer EA-DOEs have another degree of freedom, since they allow the different materials to have different profile depths. In this case it is always possible for any combination of different materials to reach $100 \%$ diffraction efficiency at two distinct wavelengths and high efficiency in between. Moreover, for multilayer and GRIN EA-DOEs, we were able to show that if the dispersion relations of the two different materials can be accurately described by a second order Cauchy series, the efficiency becomes generic in the sense that it is actually independent of the respective Cauchy coefficients. This means that the efficiency behaviour will be the same regardless of which materials are chosen. Of course, any deviation of this Cauchy relation will change the efficiency behaviour and make it material dependent. It is therefore possible to choose material combinations [12] to form a DOE with broadband efficiency above the generic curve (cf. Figure 6) generated by Eqs. (3) and (19). For disadvantageous combinations the resulting efficiency can actually be smaller than the theoretical one; an example combination is the pair PMMA and polycarbonate which only leads to minimum efficiencies of about $97 \%$.

Another insight from Section 4 is the fact that the necessary dispersion relation in Eq. (34) concerning the sub- $\lambda$-structure EA-DOE is the same as for the common depth EA-DOE Eq. (8). This becomes obvious if one remembers that both designs have the same degrees of freedom since the sub- $\lambda-$ structure EA-DOE is equivalent to the common depth EADOE due to the linear relationship Eq. (31).

One issue that has to be addressed is the thickness of the resulting EA-DOE, which can be considerably larger compared to conventional DOEs. Whereas a conventional surface relief DOE, due to its large refractive index difference between the glass or plastic material and air, yields thicknesses of only about $1 \mu \mathrm{m}$, the thicknesses of all EA-DOEs are usually more than ten times larger. This holds for the state-of-the-art multilayer DOEs as well as for the novel proposed ones. By proper choice of the materials the common depth and the multilayer EA-DOE can e.g. reach thicknesses as small as $10 \ldots 30 \mu \mathrm{m}$. The sub- $\lambda$-structure EA-DOEs may have larger thicknesses when the volume fraction difference $v_{1}\left(r_{\mathrm{b}}\right)-v_{1}\left(r_{\mathrm{a}}\right)$ is chosen to be smaller than 1 , which may be a fabrication advantage. Only GRIN EA-DOEs have significantly larger thicknesses with values in the region of $90 \ldots 230 \mu \mathrm{m}$. And even this can be exceeded if the refractive index difference is too 
small (cf. second material pair in Table 1). This problem can only be overcome by materials with larger refractive index difference as discussed earlier in Section 3.2. Additionally, there is only a small number of GRIN materials available so that their applicability is significantly reduced.

Although the scalar diffraction efficiency expressions predict always the same efficiency for any thickness, in reality, one can encounter severe losses in the efficiency if the thickness to zone width ratio becomes too large, since shadowing effects and other electromagnetic effects become significant. It is therefore advisable to look for material combinations which yield thicknesses as small as possible.

\section{ACKN OWLEDGEMENT}

We like to thank Hans-Jürgen Dobschal for his contribution to GRIN EA-DOEs as well as Wolfgang Singer for his contribution to the Tetris-design presented in Figure 19.

\section{A APPENDIX}

\section{A. 1 Phase for material dispersion with second order Cauchy relations}

In Section 2, the optical phase $\Phi(\lambda)$ of the multilayer DOE was given by Eq. (18), which is repeated for convenience:

$$
\begin{aligned}
& \Phi(\lambda)=2 \pi \times \\
& \frac{\left(\lambda_{1} n_{22}-\lambda_{2} n_{21}\right)\left(n_{1}(\lambda)-1\right)-\left(\lambda_{1} n_{12}-\lambda_{2} n_{11}\right)\left(n_{2}(\lambda)-1\right)}{\lambda\left(n_{11} n_{22}-n_{12} n_{21}\right)}
\end{aligned}
$$

with

$$
n_{i j}=a_{i}+\frac{b_{i}}{\lambda_{j}^{2}}-1, \quad i, j \in\{1,2\},
$$

based on second order Cauchy relations. To further simplify this expression, we will treat the numerator and denominator separately. The first term of the numerator can be expanded to give

$$
\begin{aligned}
& \left(\lambda_{1} n_{22}-\lambda_{2} n_{21}\right)\left(n_{1}(\lambda)-1\right)= \\
& {\left[\lambda_{1}\left(a_{2}+\frac{b_{2}}{\lambda_{2}^{2}}-1\right)-\lambda_{2}\left(a_{2}+\frac{b_{2}}{\lambda_{1}^{2}}-1\right)\right]\left(a_{1}+\frac{b_{1}}{\lambda^{2}}-1\right)=} \\
& {\left[\left(a_{2}-1\right)\left(\lambda_{1}-\lambda_{2}\right)+b_{2}\left(\frac{\lambda_{1}}{\lambda_{2}^{2}}-\frac{\lambda_{2}}{\lambda_{1}^{2}}\right)\right]\left(a_{1}-1+\frac{b_{1}}{\lambda^{2}}\right)=} \\
& \left(\lambda_{1}-\lambda_{2}\right)\left(\left(a_{2}-1\right)\left(a_{1}-1\right)+\frac{\left(a_{2}-1\right) b_{1}}{\lambda^{2}}\right)+ \\
& b_{2}\left(a_{1}-1+\frac{b_{1}}{\lambda^{2}}\right)\left(\frac{\lambda_{1}}{\lambda_{2}^{2}}-\frac{\lambda_{2}}{\lambda_{1}^{2}}\right) . \quad \text { (A.2) }
\end{aligned}
$$

Similarly, the second term can be expanded into

$$
\begin{aligned}
& \left(\lambda_{1} n_{12}-\lambda_{2} n_{11}\right)\left(n_{2}(\lambda)-1\right)= \\
& \left(\lambda_{1}-\lambda_{2}\right)\left(\left(a_{1}-1\right)\left(a_{2}-1\right)+\frac{\left(a_{1}-1\right) b_{2}}{\lambda^{2}}\right)+ \\
& b_{1}\left(a_{2}-1+\frac{b_{2}}{\lambda^{2}}\right)\left(\frac{\lambda_{1}}{\lambda_{2}^{2}}-\frac{\lambda_{2}}{\lambda_{1}^{2}}\right) .
\end{aligned}
$$

Hence, the complete numerator is given by

$$
\begin{gathered}
\left(\lambda_{1} n_{22}-\lambda_{2} n_{21}\right)\left(n_{1}(\lambda)-1\right)-\left(\lambda_{1} n_{12}-\lambda_{2} n_{11}\right)\left(n_{2}(\lambda)-1\right)= \\
\left(\lambda_{1}-\lambda_{2}\right)\left(\frac{\left(a_{2}-1\right) b_{1}}{\lambda^{2}}-\frac{\left(a_{1}-1\right) b_{2}}{\lambda^{2}}\right) \\
+\left(\left(a_{1}-1\right) b_{2}-\left(a_{2}-1\right) b_{1}\right)\left(\frac{\lambda_{1}}{\lambda_{2}^{2}}-\frac{\lambda_{2}}{\lambda_{1}^{2}}\right)= \\
\left(\left(a_{2}-1\right) b_{1}-\left(a_{1}-1\right) b_{2}\right)\left(\frac{\lambda_{1}-\lambda_{2}}{\lambda^{2}}-\frac{\lambda_{1}}{\lambda_{2}^{2}}+\frac{\lambda_{2}}{\lambda_{1}^{2}}\right) . \quad \text { (A.4) }
\end{gathered}
$$

Turning to the denominator, it can be written as

$$
\begin{aligned}
& n_{11} n_{22}-n_{12} n_{21}= \\
& \begin{aligned}
&\left(a_{1}+\frac{b_{1}}{\lambda_{1}^{2}}-1\right)\left(a_{2}+\frac{b_{2}}{\lambda_{2}^{2}}-1\right) \\
&-\left(a_{1}+\frac{b_{1}}{\lambda_{2}^{2}}-1\right)\left(a_{2}+\frac{b_{2}}{\lambda_{1}^{2}}-1\right)= \\
&\left(\left(a_{2}-1\right) b_{1}-\left(a_{1}-1\right) b_{2}\right)\left(\frac{1}{\lambda_{1}^{2}}-\frac{1}{\lambda_{2}^{2}}\right) .
\end{aligned}
\end{aligned}
$$

Putting things together, we finally obtain for $\Phi$ :

$$
\Phi(\lambda)=2 \pi \frac{\left(\frac{\lambda_{1}-\lambda_{2}}{\lambda^{2}}-\frac{\lambda_{1}}{\lambda_{2}^{2}}+\frac{\lambda_{2}}{\lambda_{1}^{2}}\right)}{\lambda\left(\frac{1}{\lambda_{1}^{2}}-\frac{1}{\lambda_{2}^{2}}\right)},
$$

which can be further cast into the final form Eq. (19) used in Section 2:

$$
\begin{aligned}
\Phi(\lambda) & =2 \pi \frac{\lambda^{2} \lambda_{1}\left(\lambda_{1}+\lambda_{2}\right)+\lambda_{2}^{2}\left(\lambda^{2}-\lambda_{1}^{2}\right)}{\lambda^{3}\left(\lambda_{1}+\lambda_{2}\right)} \\
& =2 \pi \frac{\lambda^{2} \lambda_{2}\left(\lambda_{1}+\lambda_{2}\right)+\lambda_{1}^{2}\left(\lambda^{2}-\lambda_{2}^{2}\right)}{\lambda^{3}\left(\lambda_{1}+\lambda_{2}\right)} .
\end{aligned}
$$

\section{A.2 Material relations with higher order Cauchy terms}

Now let us extend the Cauchy series of the material dispersion by a fourth order term independent of the number of materials used:

$$
n(\lambda)=a+\frac{b}{\lambda^{2}}+\frac{c}{\lambda^{4}} .
$$

In the following we represent the coefficients $a, b, c$ by the more common parameters $n_{\mathrm{d}}, v_{\mathrm{d}}$, and $\theta_{\mathrm{g}, \mathrm{F}}$, which are, respectively, refractive index, Abbe number, and relative partial dispersion. The latter is defined as

$$
\theta_{\mathrm{g}, \mathrm{F}}=\frac{n\left(\lambda_{\mathrm{g}}\right)-n\left(\lambda_{\mathrm{F}}\right)}{n\left(\lambda_{\mathrm{F}}\right)-n\left(\lambda_{\mathrm{C}}\right)}
$$

with the wavelengths given by

$$
\begin{aligned}
& \lambda_{\mathrm{g}}=422.67 \mathrm{~nm}, \lambda_{\mathrm{F}}=486.13 \mathrm{~nm}, \\
& \lambda_{\mathrm{d}}=587.56 \mathrm{~nm}, \lambda_{\mathrm{C}}=656.28 \mathrm{~nm} .
\end{aligned}
$$

Using the Abbe number

$$
v_{\mathrm{d}}=\frac{n\left(\lambda_{\mathrm{d}}\right)-1}{n\left(\lambda_{\mathrm{F}}\right)-n\left(\lambda_{\mathrm{C}}\right)},
$$


we can rewrite the relative partial dispersion as

$$
\theta_{\mathrm{g}, \mathrm{F}}=\left(n\left(\lambda_{\mathrm{g}}\right)-n\left(\lambda_{\mathrm{F}}\right)\right) \frac{v_{\mathrm{d}}}{n_{\mathrm{d}}-1}
$$

Using the Cauchy series (A.7) we obtain for the Abbe number

$$
v_{\mathrm{d}}=\frac{n_{\mathrm{d}}-1}{b\left(\lambda_{\mathrm{F}}^{-2}-\lambda_{\mathrm{C}}^{-2}\right)+c\left(\lambda_{\mathrm{F}}^{-4}-\lambda_{\mathrm{C}}^{-4}\right)}
$$

and for the relative partial dispersion

$$
\theta_{\mathrm{g}, \mathrm{F}}=\left(b\left(\lambda_{\mathrm{g}}^{-2}-\lambda_{\mathrm{F}}^{-2}\right)+c\left(\lambda_{\mathrm{g}}^{-4}-\lambda_{\mathrm{F}}^{-4}\right)\right) \frac{\nu_{\mathrm{d}}}{n_{\mathrm{d}}-1} .
$$

Using the notation

$$
\begin{aligned}
& \lambda_{\mathrm{g}, \mathrm{F}}^{2}=\lambda_{\mathrm{g}}^{-2}-\lambda_{\mathrm{F}}^{-2}, \lambda_{\mathrm{g}, \mathrm{F}}^{4}=\lambda_{\mathrm{g}}^{-4}-\lambda_{\mathrm{F}}^{-4}, \\
& \lambda_{\mathrm{F}, \mathrm{C}}^{2}=\lambda_{\mathrm{F}}^{-2}-\lambda_{\mathrm{C}}^{-2}, \lambda_{\mathrm{F}, \mathrm{C}}^{4}=\lambda_{\mathrm{F}}^{-4}-\lambda_{\mathrm{C}}^{-4},
\end{aligned}
$$

we get the following relations between the coefficients of the Cauchy series and the material parameters:

$$
\begin{aligned}
& b \lambda_{\mathrm{F}, \mathrm{C}}^{2}+c \lambda_{\mathrm{F}, \mathrm{C}}^{4}=\frac{v_{\mathrm{d}}}{n_{\mathrm{d}}-1}, \\
& b \lambda_{\mathrm{g}, \mathrm{F}}^{2}+c \lambda_{\mathrm{g}, \mathrm{F}}^{4}=\left(n_{\mathrm{d}}-1\right) \frac{\theta_{\mathrm{g}, \mathrm{F}}}{v_{\mathrm{d}}},
\end{aligned}
$$

which look simpler in matrix notation:

$$
\left(\begin{array}{ll}
\lambda_{\mathrm{F}, \mathrm{C}}^{2} & \lambda_{\mathrm{F}, \mathrm{C}}^{4} \\
\lambda_{\mathrm{g}, \mathrm{F}}^{2} & \lambda_{\mathrm{g}, \mathrm{F}}^{4}
\end{array}\right)\left(\begin{array}{l}
b \\
c
\end{array}\right)=\left(\begin{array}{c}
\frac{v_{\mathrm{d}}}{n_{\mathrm{d}}-1} \\
\frac{n_{\mathrm{d}}-1}{v_{\mathrm{d}}} \theta_{\mathrm{g}, \mathrm{F}}
\end{array}\right)
$$

Solving the linear system by matrix inversion we finally obtain:

$$
\left(\begin{array}{l}
b \\
c
\end{array}\right)=\frac{1}{\lambda_{\mathrm{F}, \mathrm{C}}^{2} \lambda_{\mathrm{g}, \mathrm{F}}^{4}-\lambda_{\mathrm{F}, \mathrm{C}}^{4} \lambda_{\mathrm{g}, \mathrm{F}}^{2}}\left(\begin{array}{cc}
\lambda_{\mathrm{g}, \mathrm{F}}^{4} & -\lambda_{\mathrm{F}, \mathrm{C}}^{4} \\
-\lambda_{\mathrm{g}, \mathrm{F}}^{2} & \lambda_{\mathrm{F}, \mathrm{C}}^{2}
\end{array}\right)\left(\begin{array}{c}
\frac{v_{\mathrm{d}}}{n_{\mathrm{d}}-1} \\
\frac{n_{\mathrm{d}}-1}{v_{\mathrm{d}}} \theta_{\mathrm{g}, \mathrm{F}}
\end{array}\right) .
$$

Hence, the coefficients $b$ and $c$ can now be calculated for a given Abbe number $v_{\mathrm{d}}$ and relative partial dispersion $\theta_{\mathrm{g}, \mathrm{F}}$. Finally, the remaining coefficient $a$ results from

$$
a=n_{\mathrm{d}}-\frac{b}{\lambda_{\mathrm{d}}^{2}}-\frac{c}{\lambda_{\mathrm{d}}^{4}}
$$

These relations can now be used as a starting point for the representation of the phase $\Phi(\lambda)$ with higher order Cauchy terms using coefficients $a, b, c$ or relations $n_{\mathrm{d}}, v_{\mathrm{d}}$, and $\theta_{\mathrm{g}, \mathrm{F}}$.

\section{B APPENDIX}

In this appendix it is shown that the nonlinear Eqs. (29) and (30) can be approximated by the same linear Eq. (31) used in Section 4 to sufficient accuracy. Without any loss of generality, we are assuming $n_{1}>n_{2}$ as in the whole publication, so that

$$
n_{1}=n_{2}+\Delta n, \quad \text { with } \Delta n=n_{1}-n_{2} \ll 1 .
$$

\section{B.1 TE-polarization}

In TE-polarization, the effective refractive index $n_{\text {eff }}$ can be calculated by the relation [36]

$$
n_{\mathrm{eff}}=\sqrt{v_{1} n_{1}^{2}+\left(1-v_{1}\right) n_{2}^{2}}=\sqrt{v_{1}\left(n_{2}+\Delta n\right)^{2}+\left(1-v_{1}\right) n_{2}^{2}} .
$$

Expanded and simplified one obtains:

$$
n_{\mathrm{eff}}=\sqrt{n_{2}^{2}\left\{1+2 v_{1} \frac{\Delta n}{n_{2}}+\left(\frac{v_{1} \Delta n}{n_{2}}\right)^{2}\right\}} .
$$

Omitting terms of order $(\Delta n)^{2}$ and using the first two terms of the series expansion of the square root, one obtains:

$$
n_{\mathrm{eff}} \approx n_{2}\left(1+v_{1} \frac{\Delta n}{n_{2}}\right)
$$

Using $\Delta n=n_{1}-n_{2}$, this can be rewritten as

$$
n_{\mathrm{eff}} \approx v_{1} n_{1}+\left(1-v_{1}\right) n_{2}
$$

which is the same linear relation Eq. (31) used in Section 4.

Now, it is additionally shown that the difference between the nonlinear Eqs. (B.2) and the linear Eqs. (B.5) is sufficiently small. This difference is a function of the volume fraction $v_{1}$ :

$$
\Delta\left(v_{1}\right)=\sqrt{v_{1} n_{1}^{2}+\left(1-v_{1}\right) n_{2}^{2}}-v_{1} n_{1}-\left(1-v_{1}\right) n_{2} .
$$

To determine the maximum value of difference $\Delta\left(v_{1}\right)$, its first derivative is calculated:

$$
0=\frac{\mathrm{d}}{\mathrm{d} v_{1}} \Delta\left(v_{1}\right)=\frac{n_{1}^{2}-n_{2}^{2}}{2 \sqrt{v_{1} n_{1}^{2}+\left(1-v_{1}\right) n_{2}^{2}}}-n_{1}+n_{2} .
$$

Solving this equation for $v_{1}$ yields the value of $v_{1 \text {,max }}$ where $\Delta\left(v_{1}\right)$ has its maximum value. One obtains

$$
v_{1, \max }=\frac{n_{1}+3 n_{2}}{4\left(n_{1}+n_{2}\right)} .
$$

Inserting in Eq. (B.6), the maximum value for the difference is

$$
\Delta\left(v_{1, \max }\right)=\frac{\left(n_{1}-n_{2}\right)^{2}}{4\left(n_{1}+n_{2}\right)} .
$$

Thus, the maximal expected mismatch $\Delta\left(v_{1, \max }\right)$ is proportional to the square of $n_{1}-n_{2}$ which is typically smaller than $0.1^{2}=0.01$. The denominator in Eq. (B.9) has a typical value of 12. Therefore, for the effective refractive index Eq. (B.5) an error of

$$
\Delta\left(v_{1, \max }\right) \leq 0.001
$$

is expected which is small enough to be neglected. Hence, it is justified to replace the nonlinear Eq. (29) for the effective refractive index by the linear Eq. (31).

\section{B.2 TM-polarization}

Now, in TM-polarization, the effective refractive index $n_{\text {eff }}$ can be calculated by the relation [36]

$$
n_{\mathrm{eff}}=\frac{1}{\sqrt{\frac{v_{1}}{n_{1}^{2}}+\frac{\left(1-v_{1}\right)}{n_{2}^{2}}}}=\frac{1}{\sqrt{\frac{v_{1}}{\left(n_{2}+\Delta n\right)^{2}}+\frac{\left(1-v_{1}\right)}{n_{2}^{2}}}} .
$$


This is equivalent to

$$
n_{\mathrm{eff}}=\frac{1}{\sqrt{\frac{1}{n_{2}^{2}}\left\{\frac{v_{1}}{\left(1+\frac{\Delta n}{n_{2}}\right)^{2}}+\left(1-v_{1}\right)\right\}}}
$$

Using the first two terms of the series expansion of $(1+x)^{-2}$ for the denominator of the first term in the brackets under the square root, this can be approximated by

$$
n_{\mathrm{eff}} \approx \frac{n_{2}}{\sqrt{1-\frac{2 v_{1} \Delta n}{n_{2}}}}
$$

Again, utilizing only the first two terms of $(1-x)^{-\frac{1}{2}}$ series expansion, it results in

$$
n_{\mathrm{eff}} \approx n_{2}\left(1+v_{1} \frac{\Delta n}{n_{2}}\right)
$$

With $\Delta n=n_{1}-n_{2}$, this can be rewritten into the linear relation Eq. (31) used in Section 4:

$$
n_{\mathrm{eff}} \approx v_{1} n_{1}+\left(1-v_{1}\right) n_{2}
$$

Also for TM-polarization, in the following it is shown that the difference between the nonlinear Eqs. (B.11) and the linear Eqs. (B.15) is sufficiently small. Again, this difference is a function of the volume fraction $v_{1}$ :

$$
\Delta\left(v_{1}\right)=\frac{1}{\sqrt{\frac{v_{1}}{n_{1}^{2}}+\frac{\left(1-v_{1}\right)}{n_{2}^{2}}}}-v_{1} n_{1}-\left(1-v_{1}\right) n_{2} .
$$

The first derivative of difference $\Delta\left(v_{1}\right)$ is given by:

$0=\frac{\mathrm{d}}{\mathrm{d} v_{1}} \Delta\left(v_{1}\right)=-\frac{1}{2} \frac{1}{\sqrt{\left(\frac{v_{1}}{n_{1}^{2}}+\frac{\left(1-v_{1}\right)}{n_{2}^{2}}\right)^{3}}} \cdot\left(\frac{1}{n_{1}^{2}}-\frac{1}{n_{2}^{2}}\right)-n_{1}+n_{2}$.

Solving this equation for $v_{1}$ yields the value of $v_{1 \text {,max }}$ where $\Delta\left(v_{1}\right)$ has its maximum value. The only real zero is

$$
v_{1, \max }=\frac{\left[\frac{1}{2}\left(1+\frac{n_{2}}{n_{1}}\right) \frac{n_{2}}{n_{1}}\right]^{2 / 3}-1}{\frac{n_{2}^{2}}{n_{1}^{2}}-1} .
$$

The maximum value for the expected mismatch $\Delta\left(v_{1, \max }\right)$ of the effective refractive index Eq. (B.15) can be calculated by inserting this expression into Eq. (B.16). For typical values of refractive index and refractive index difference with e.g. $n_{1}=$ $1.5, n_{1}-n_{2}=0.1$, respectively, it can be estimated:

$$
\left|\Delta\left(v_{1, \max }\right)\right| \leq 0.0025
$$

This value is small enough to be neglected in the TMpolarization case.

The final result is, that in both polarization cases, the nonlinear relations Eqs. (29) and (30) can be approximated by the same linear Eq. (31) to sufficient accuracy.

\section{References}

[1] T. Stone, N. George, "Hybrid diffractive-refractive lenses and achromats" Appl. Optics 27, 2960-2971 (1988).

[2] R. Brunner, R. Steiner, K. Rudolf, and H. J. Dobschal, "DiffractiveRefractive Hybrid Microscope Objective for $193 \mathrm{~nm}$ Inspection Systems" Proc. SPIE 5177, 9-15 (2003).

[3] R. Brunner, R. Steiner, H. J. Dobschal, D. Martin, M. Burkhardt, and M. Helgert, "New Solution to Realize Complex Optical Systems by a Combination of Diffractive and Refractive Optical Components" Proc. SPIE 5183, 47-55 (2003).

[4] R. Brunner, A. Menck, R. Steiner, G. Buchda, S. Weissenberg, U. Horn, and A. Zibold, "Immersion Mask Inspection with Hybrid Microscopic System at 193 nm" Proc. SPIE 5567, 887-893 (2004).

[5] H. J. Dobschal, "Two examples for the effective use of hybrid optics" EOS Top. Meet. Diffractive Optics, 84-85 (2007).

[6] C. Sauvan, P. Lalanne, and M.-Si L. Lee, "Broadband blazing with artificial dielectrics" Opt. Lett. 29, 1593-1595 (2004).

[7] E. Popov, B. Bozhkov, and M. Neviere, "Almost Perfect Blazing by Photonic Crystal Rod Gratings" Appl. Optics 40, 2417-2422 (2001).

[8] S. M. Ebstein, "Nearly index-matched optics for aspherical, diffractive, and achromatic-phase diffractive elements" Opt. Lett. 21, 1454-1456 (1996).

[9] Y. Arieli, S. Ozeri, and N. Eisenberg, "Design of a diffractive optical element for wide spectral bandwidth" Opt. Lett. 23, 823-824 (1998).

[10] T. Nakai, "Diffractive optical element" European Patent Specification EP 965864 B1 (1998).

[11] T. Nakai, and H. Ogawa, "Research on multi-layer diffractive optical elements and their application to camera lenses" OSA Tech. Dig. of DOMO Conf., Rochester, 5-7 (2002).

[12] T. Nakai, "Diffractive optical element and optical system having the same" European Patent Specification EP 898182 B1 (1997).

[13] A. Schilling, K. J. Weible, and H. P. Herzig, "Diffractive structures with high, wavelength independent efficiency" EOS Top. Meet. Dig. Ser. 22, 16-17 (1999).

[14] K. J. Weible, A. Schilling, H. P. Herzig, and D. Lobb, "Achromatization of the diffraction efficiency of diffractive optical elements" Proc. SPIE 3749, 378-379 (1999).

[15] A. Schilling and H. P. Herzig, "Optical System Design Using Microoptics" in Encyclopedia of Optical Engineering, R. G. Driggers, ed., 1830-1842 (Marcel Dekker Inc., New York, 2003).

[16] B. Achtner, F. 0. Karutz, M. Pollmann, and M. Seeßelberg, "Videobrille für das Kino unterwegs" Photonik 40, 40-43 (2008).

[17] M. D. Missing and G. Michael Morris, "Diffractive optics applied to eyepieces design" Appl. Optics 34, 2452-2461 (1995).

[18] 0. Sandfuchs, D. Pätz, S. Sinzinger, A. Pesch, and R. Brunner, "Analysis of the influence of the passive facet of blazed transmission gratings in the intermediate diffraction regime" J. Opt. Soc. Am. A 25, (2008).

[19] D. A. Buralli, G. M. Morris, and J. R. Rogers, "Optical performance of holographic kinoforms" Appl. Optics 28, 976-983 (1989).

[20] B. H. Kleemann, A. Mitreiter, and F. Wyrowski, "Integral equation method with parametrization of grating profile - Theory and experiments" J. Mod. Optic. 43, 1323-1349 (1996).

[21] A. Rathsfeld, C. Schmidt, and B. H. Kleemann, “On a Fast Integral Equation Method for Diffraction Gratings" Commun. Comput. Phys. 
1, 984-1009 (2006).

[22] H. Ukuda, "Optical material, and optical element, optical system and laminated diffractive optical element using it", European Patent Application EP 1394574 (2003).

[23] W. Stork, N. Streibl, H. Haidner, and P. Kipfer, "Artificial distributed-index media fabricated by zero-order gratings" Opt. Lett. 16, 1921-1923 (1991).

[24] P. Lalanne, S. Astilean, P. Chavel, E. Cambril, and H. Launois, "Design and fabrication of blazed binary diffractive elements with sampling periods smaller than the structural cutoff" J. Opt. Soc. Am. A 16, 1143-1156 (1999).

[25] J. N. Mait, D. W. Prather, and M. S. Mirotznik, "Design of binary subwavelength diffractive lenses by use of zeroth-order effectivemedium theory" J. Opt. Soc. Am. A 16, 1157-1167 (1999).

[26] Mane-Si Laure Lee, P. Lalanne, P. Chavel, and E. Cambril, "Imaging with blazed-binary diffractive elements" Proc. SPIE on Physics, Theory, and Applications of Periodic Structures in Optics, P. Lalanne, ed., 4438, 62-68 (2001).

[27] B. H. Kleemann, J. Ruoff, and R. Arnold, "Area-coded effective medium structures, a new type of grating design" Opt. Lett. 30, 1617-1619 (2005).

[28] Information from GRINTECH AG in 2004.

[29] R. P. Salmio, J. Saarinen, J. Turunen, and A. Tervonen, "Gradedindex diffractive structures fabricated by thermal ion exchange" Appl. Optics 36, 2048-2057 (1997).

[30] T. Vahrenkamp, H. Kreitlow, H. Schütte, and C. Thoma, “DOE aus Glas für den Nd:YAG-Laser" Photonik 3, 6-8 (2002).

[31] J. Teteris, "Holographic recording in amorphous chalcogenide thin films" Current Opinion in Solid State and Material Science 7, 127134 (2003).

[32] T. Buffeteau, F. Lagugnè Labarthet, C. Sourisseau, S. Kostromine, and T. Bieringer, "Biaxial orientation induced in a photoaddressable azopolymer thin film as evidenced by polarized UVVisible, infrared, and Raman spectra" Macromolecules 37, 28802889 (2004).

[33] R. Hagen and T. Bieringer, "Photoaddressable polymers for data storage" Advanced Mat. 13 1805-1810 (2001)

[34] J. M. Tsui, C. Thompson, V. Mehta, J. M. Roth, V. I. Smirnov, and L. B. Glebov, "Coupled-wave analysis of apodized volume gratings" Opt. Express 12, 6642-6653 (2004).

[35] J. Yeh, A. Harton, and K. Wyatt, "Reliability study of holographic optical elements made with DuPont photopolymer" Appl. Optics 37, 6270-6274 (1998).

[36] S. M. Rytov, "Electromagnetic properties of a finely stratified medium" Sov. Phys. JETP-USSR 2 466-475, (1956).

[37] http://www.texloc.com/closet/cl_refractiveindex.html

[38] M. Colburn, S. Johnson, M. Stewart, S. Damle, T. Bailey, B. J. Choi, M. Wedlake, T. Michaelson, S. V. Sreenivasan, C. G. Willson, “Step and Flash Imprint Lithography: A new approach to high-resolution patterning" Proc. SPIE on Microlithography 3676, 379-389 (1999).

[39] http://www.cargille.com

[40] D. Mund, K. M. Hammerl, "Building up diffractive optics by structured glass coatings" Patent Application Publication WO $2005121842 \mathrm{~A} 1$

[41] http://en.wikipedia.org/wiki/Tetris 